University of Nebraska - Lincoln

DigitalCommons@University of Nebraska - Lincoln

20th \& 21st Century French and Francophone Modern Languages and Literatures, Department Studies International Colloquium

\title{
Intégration du genre dans la gestion des ressources en eau au Maroc (Nord-Ouest d'Afrique)
}

El-Mostafa Mili

Follow this and additional works at: https://digitalcommons.unl.edu/ffsc2020

Part of the Comparative Literature Commons, French and Francophone Literature Commons, and the Other French and Francophone Language and Literature Commons

This Presentation is brought to you for free and open access by the Modern Languages and Literatures, Department of at DigitalCommons@University of Nebraska - Lincoln. It has been accepted for inclusion in 20th \& 21st Century French and Francophone Studies International Colloquium by an authorized administrator of DigitalCommons@University of Nebraska - Lincoln. 

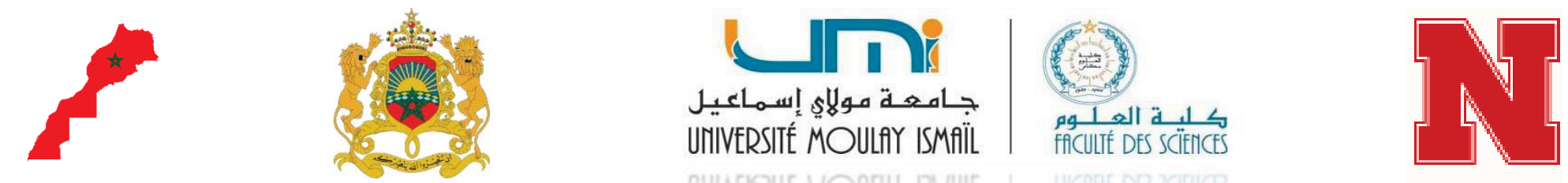

\section{PANEL}

$1 \mathrm{E})$ Le Maroc: pour une sauvegarde du patrimoine naturel et culturel, Florina Matu (St. Edward's University)

NEBRASKA, 26 Mars 2020

\section{1) Intégration du genre dans la gestion des ressources en eau au Maroc (Nord- Ouest d'Afrique)}

Pr. El-Mostafa MILI

mili.elmostafa3@gmail.com

Laboratoire Géo-Ingénierie et Environnement, Département de Géologie, Faculté des Sciences, Université Moulay Ismaïl, Meknès 


\section{INTRODUCTION}

Au Maroc 92\% des individus âgés de plus de 5 ans ont un téléphone (2018), alors que $80 \%$ de Marocains ont accès à l'eau potable et $38 \%$ disposent d'un accès aux installations d'assainissement améliorées (2017) !

Le Maroc a une grande expérience en matière de développement de l'offre des ressources en eau. Malgré cela, les problèmes de pénurie d'eau sont devenus une réalité.

Malheureusement, les intérêts particuliers et la corruption généralisée entre les gouvernements et les entrepreneurs privés génèrent trop souvent une situation déséquilibrée.

Est-ce que cela a pour cause la mauvaise gestion des ressources en eau?

L'objectif de ce travail est de donner l'état de l'intégration du genre dans la GIRE et d'y montrer son importance. 


\section{SITUATION}

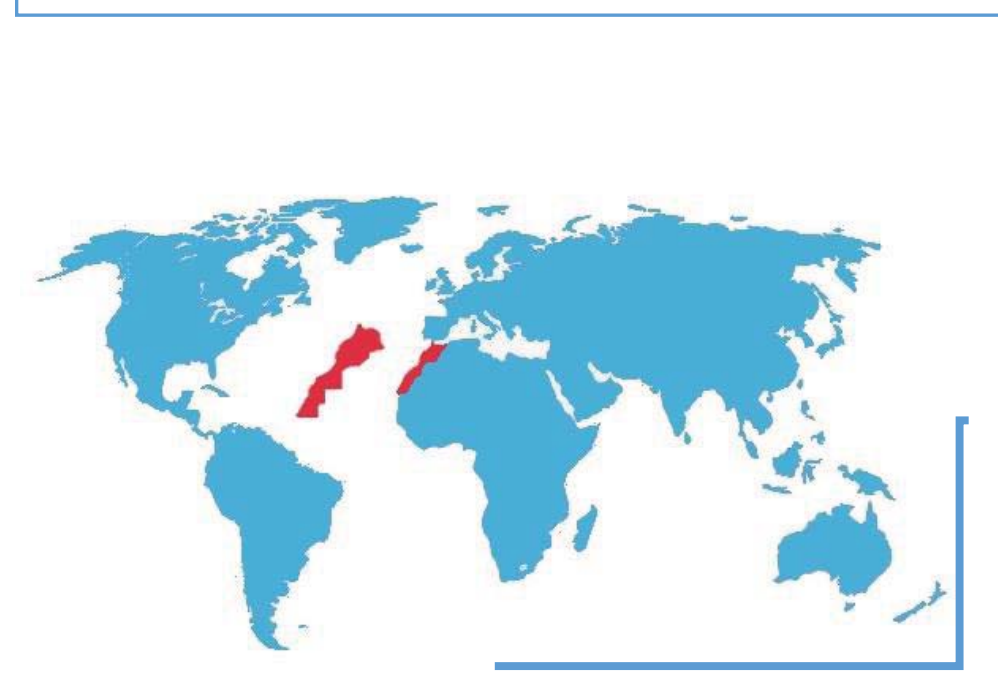

Le Maroc est situé au NW de l'Afrique, il est limité au $\mathrm{N}$ par $512 \mathrm{~km}$ de la mer méditerranée, à l'ouest par 2934 $\mathrm{km}$ d'océan atlantique.

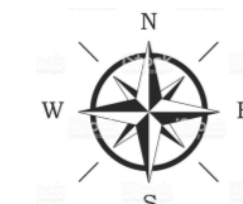

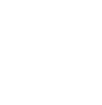




\section{Organigramme synthétique de la}

Gestion globale de l'eau

- Compromis

- Arbitrer

- aménager

\begin{tabular}{l|l}
\hline & Socit \\
Drit \\
Insti
\end{tabular}

Politique

conomie

Sociologie

Institutions

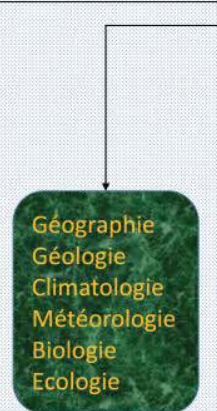

Ressources

(Mobilisables et utilisables) = Offres

Flux

-Stock

Hydro-mécanique Hydrologie

\section{-Ecoulements}

Ressources en eau

naturelles
MILIEU

et

Social

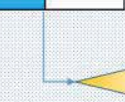

Confrontation

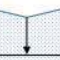

Aménagement des

eaux

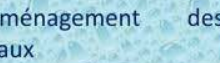

tilisation des eaux
Demandes d'eau

IN SITU/A PRELEVER

-Quantité -Prix (de revient, d'achat)

-Qualité

GESTION

DES EAUX

Technologi

Génie civil : Mécanique

Génie chimique

Electrotechnique

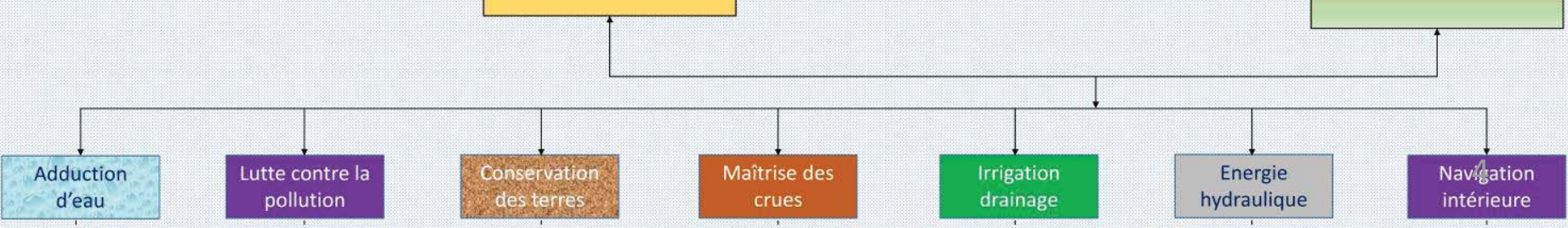




\section{INTÉGRATION DU GENRE DANS LA GIRE}

Le genre peut agir largement dans les thèmes sectoriels de l'eau suivants :

1) gouvernance et gestion des ressources en eau ;

2) eau et pauvreté ;

3) installations sanitaires et hygiène ;

4) distribution d'eau à usage domestique ;

5) eau à usage agricole et irrigation ;

6) eau et environnement ;

7) gestion des zones côtières ;

8) variation climatique et catastrophes reliées à l'eau ;

9) renforcement des capacités ;

10) planification et outils du genre dans le secteur de l'eau ;

11) initiatives budgétaires pour l'équité entre les sexes dans les secteurs de l'eau. 


\section{INTÉGRATION DU GENRE DANS LA GIRE}

La gestion intégrée de la ressource en eau (GIRE) est l'application des principes du développement durable au secteur de l'eau. Dans un cadre territorial cohérent, la GIRE vise à intégrer les multiples parties prenantes, usages et enjeux concurrents, dont la préservation environnementale, afin d'assurer la pérennité des ressources en eau. Pour prendre en compte les nombreuses interactions et interdépendances entre ces composantes, la GIRE prône une gouvernance participative. La GIRE exige une conscience genre en développant la participation entière et efficace des femmes à tous les niveaux de la prise de décision. 


\section{INTÉGRATION DU GENRE DANS LA GIRE}

Dans le GIRE, il faudra prendre en compte la manière dont les différentes sociétés affectent des rôles sociaux, économiques et culturels particuliers aux hommes et aux femmes. II y a une synergie importante entre l'équité genre et la gestion durable de l'eau. Faire participer les hommes et les femmes dans les rôles influents à tous les niveaux de la gestion de l'eau peut accélérer la réalisation de la pérennité. La gestion de l'eau de manière intégrée et durable contribue significativement à l'équité genre en améliorant l'accès des femmes et des hommes à l'eau et aux services connexes à l'eau pour la satisfaction de leurs besoins essentiels. 
Cet organigramme montre les rôles et les responsabilités des instances et établissements publics et privés dans le secteur de l'eau très fragmenté en raison de la diversité des ses utilisations (Domestique, Agricole, Industrielle et commerciale, Energétique, Municipale...). II en résulte que toute décision prise aura un impact sur tous les autres secteurs économiques du pays.

ORGANISATION DU SECTEUR DE L'EAU AU MAROC

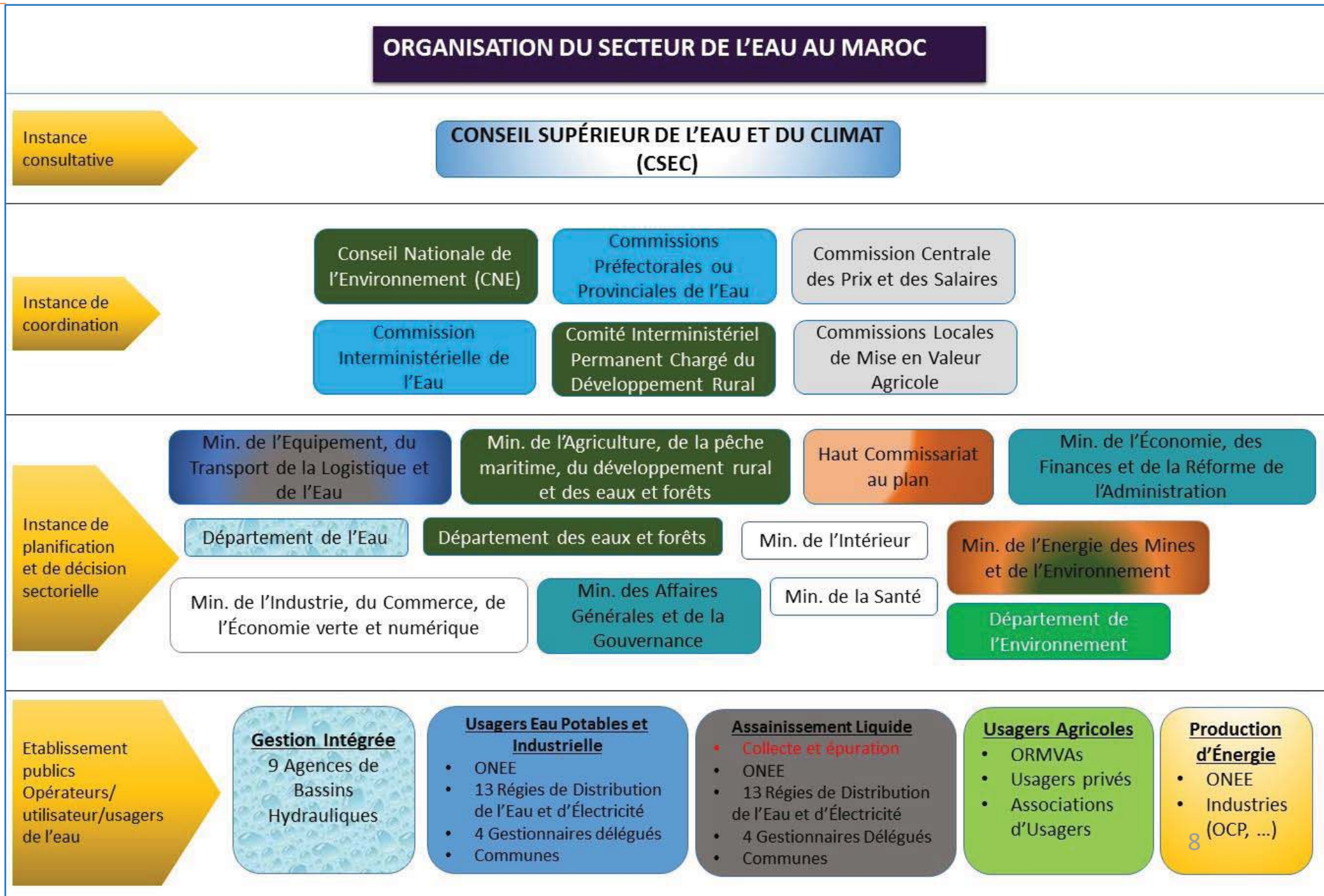




\section{GOUVERNANCE DE L'EAU AU MAROC}

La relation entre les différents départements ministériels et leurs attributions dans la planification, l'inventaire, le financement, la mobilisation des ressources en eau, l'irrigation, I'AEPI, I'hydroélectricité et la maintenance des ouvrages publics donne l'ordre d'attributions suivant :

1 Eau

2 Agriculture

3 Energie et Mines

4 Intérieur

5 Environnement, Santé

6 Eaux et Forêts, Commerce et Industrie

7 Habitat

8 Finances, Equipement, Aménagement du territoire

9 Justice, Plan, Tourisme, Enseignement, Affaires culturelles, Défense nationale, Habous (institution du droit musulman)

On en déduit d'abord que le secteur de l'eau offre plusieurs opportunités d'emploi et ensuite que les Départements les plus importants dans ce secteur vital sont l'eau et l'agriculture. 


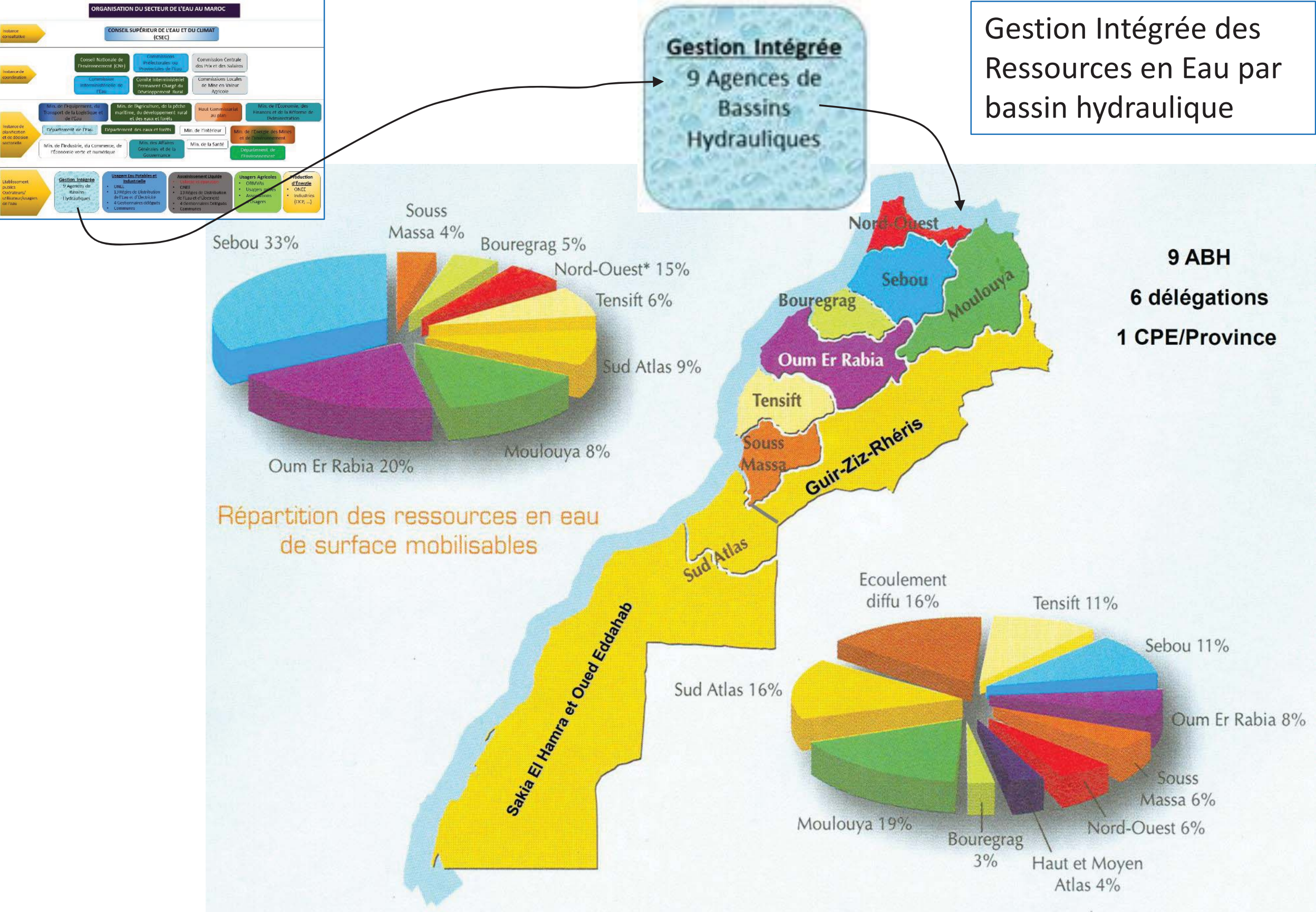

Sebou 33\% Massa $4 \%$

Bouregrag 5\% Agences de

Bassins bassin hydraulique

Répartition des ressources en eau de surface mobilisables

Répartition des ressources en eau 10 https://www.oecd.org/mena/governance/43316384.pdf souterraine mobilisables 


\section{IMPACTS DU RÉCHAUFFEMENT CLIMATIQUE}

Les prévisions du GIEC en 2100, montrent que l'Afrique est parmi les continents les plus menacés par les impacts du réchauffement climatique à savoir le problème de la pénurie d'eau, d'insécurité alimentaire, de maladies.

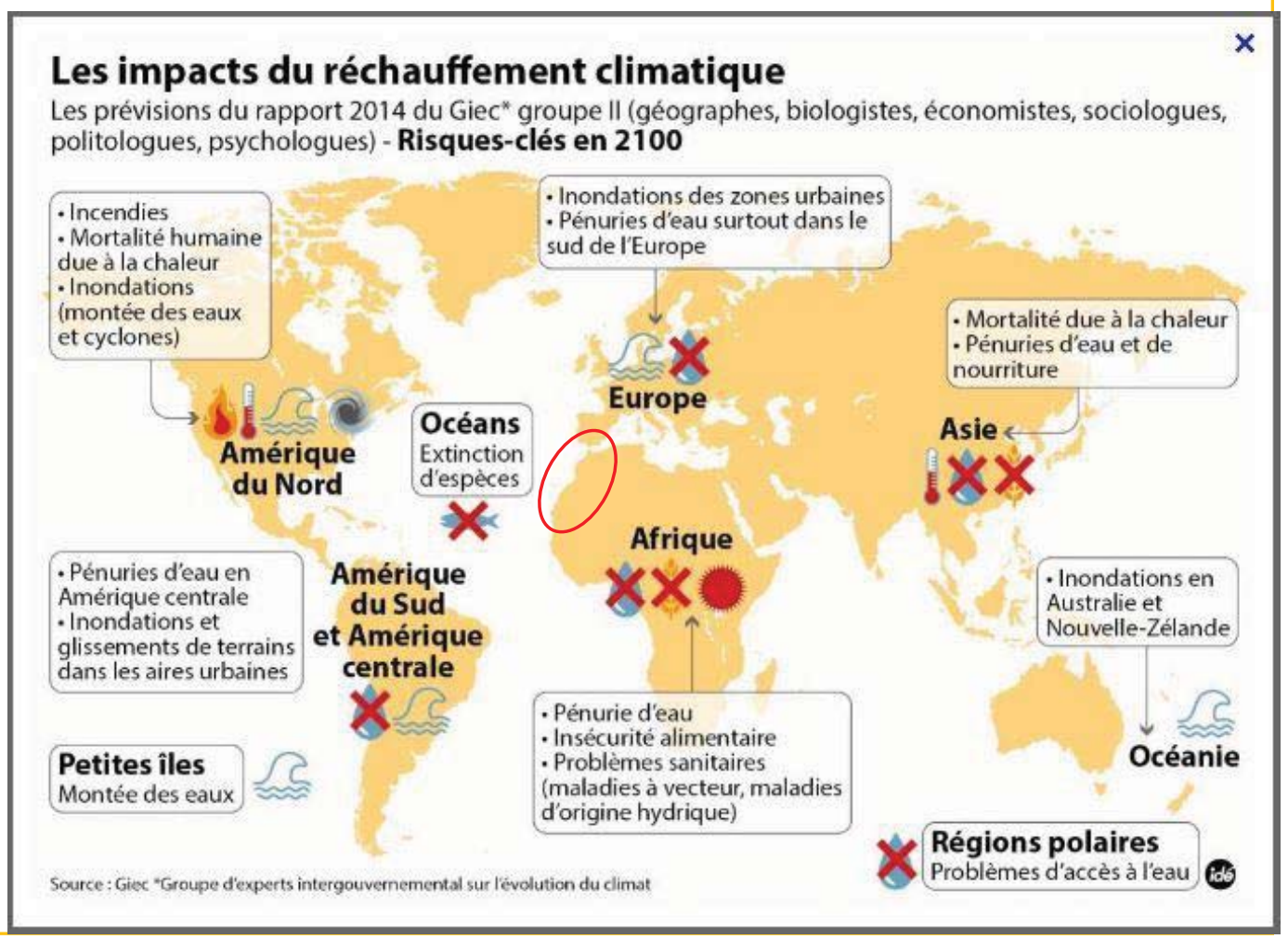




\section{PERFORMANCE DU MAROC EN MATIÈRE DE PARITÉ}

- Le Maroc est classé en rang 136 pour la parité hommesfemmes au classement général comptant 144 pays, dans les domaines du travail, de l'éducation, de la santé et de la politique.
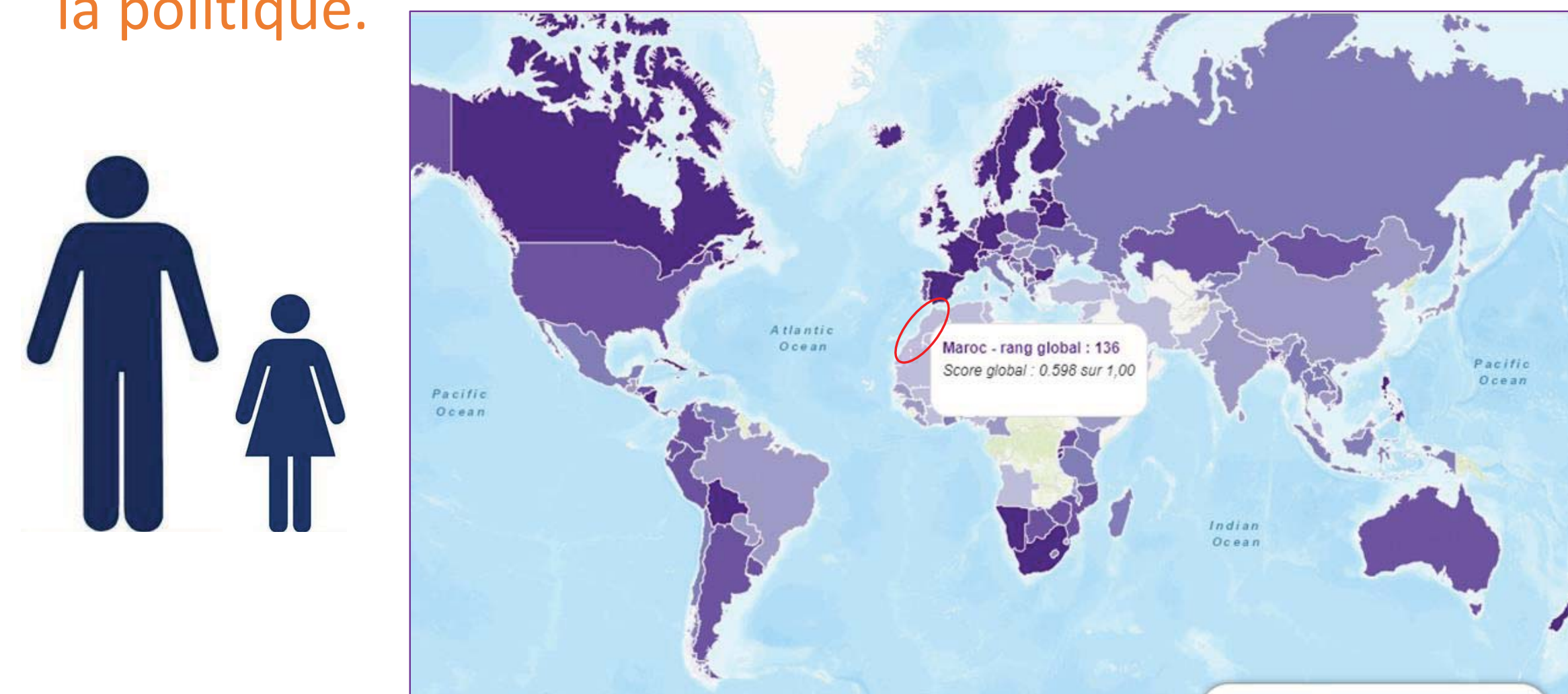

2017 


\section{ÉNERGIES PROPRES}

L'alimentation des usines de dessalement par énergie électrique diminuera le coût du $\mathrm{m}^{3}$ d'eau douce. Au Maroc en 2017, la production électrique des énergies renouvelables reste encore assez faible sachant qu'elle dépasse $50 \%$ dans les $1^{\text {er }}$ rangs.

PRODUCTION ÉLECTRIQUE ISSUE DES ÉNERGIES RENOUVELABLES EN 2017

PART DES ÉNERGIES RENOUVELABLES HORS

HYDROÉLECTRICITÉ

(\% de la production électrique)

$\geq 50$

$40-50$

$30-40$

$20-30$

$10-20$

- 5-10

$0-5$

0

Données indisponibles

(C) ATLASOCIO.COM

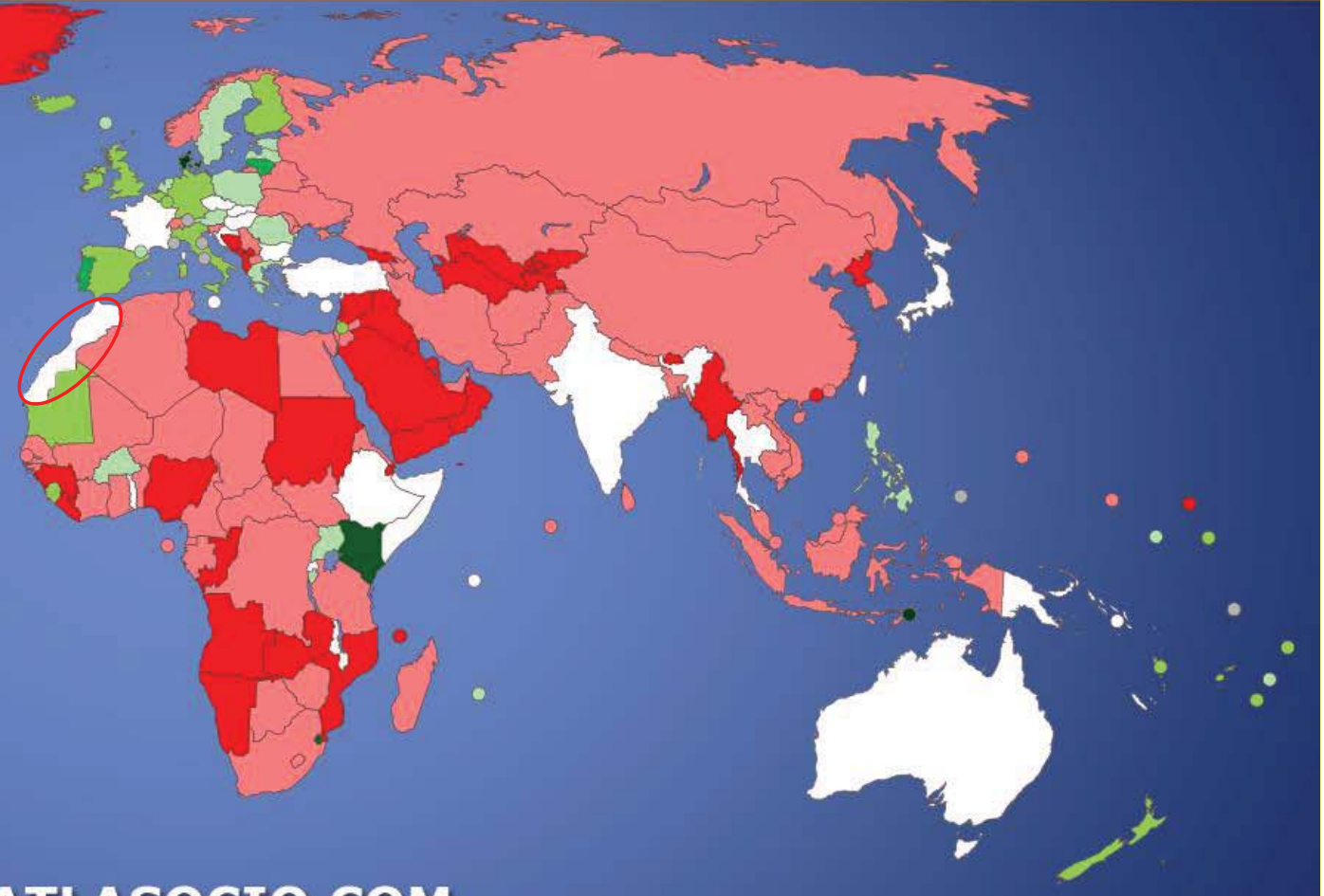




\section{RÉDUCTION DE LA DISPONIBILITÉ EN EAU}

La disponibilité en eau du Maroc en 1950 correspondait à une situation hydrique basse, à partir de 1995, la situation est catastrophiquement basse. En 2040, le Maroc sera en situation de stress hydrique élevé à extrêmement élevé.

1950

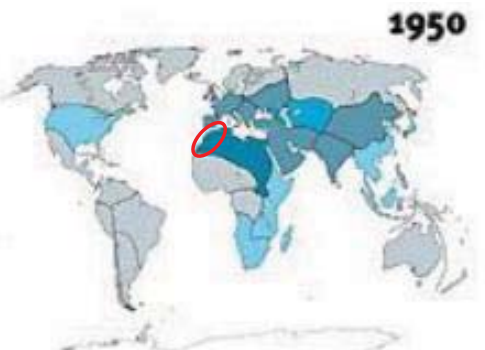

Disponibilité en eau (en milliers de $\mathrm{m}^{3}$ par personne et par an) $<\mathbf{1}, \mathbf{0}=$ catastrophiquement basse $1.1-2$ = basse

$5.1-10=$ moyenne
10.1 - 20 = élevée

$20=$ trés élevée
Stress hydrique, projections en 2040

2025

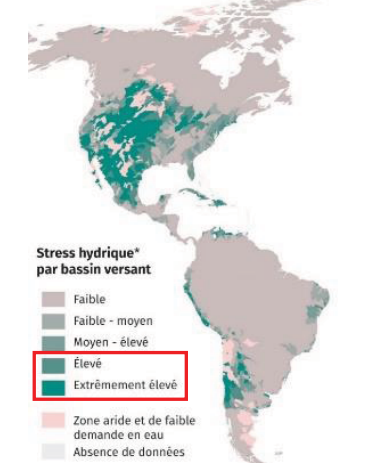

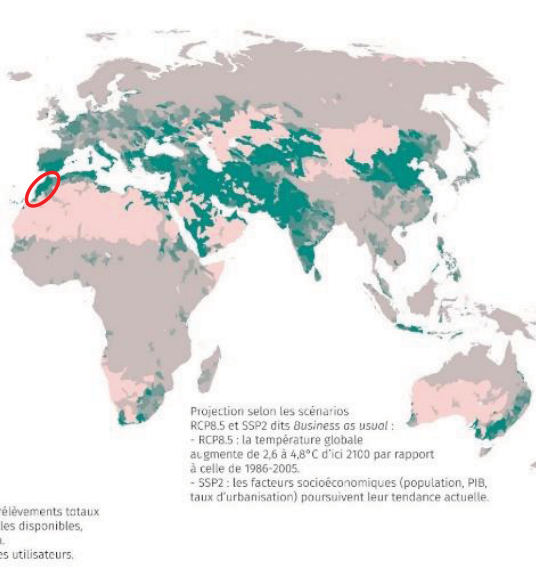




\section{RARÉFACTION DES RESSOURCES EN EAU}

Dans le calcul du ratio, la ressource en eau naturelle renouvelable est supposée constante. Or la Direction de la Météorologie Nationale prévoit une régression de $20 \%$ en moyenne des précipitations d'ici la fin du siècle à la faveur de l'évaporation et de l'évapotranspiration qui augmentent avec la température du réchauffement du climat.

II y a aussi le problème d'évaporation des barrages qui se produit au rythme de 20 litres par jour et par mètre carré, en moyenne, durant les mois chauds. 


\section{Baseline Water Stress}

Low ( $<10 \%$ )

Low to medium (10-20\%)

Medium to high (20-40\%)

- High (40-80\%)

Extremely high $(>80 \%)$

Arid \& low water use

No data

\section{Definition}

Baseline water stress measures the ratio of total annual water withdrawals to total available annual renewable supply. accounting for upstream consumptive use Higher values indicate more competition among users

\section{Sources: WRI Aqueduct:2014, FAO} AQUASTAT 2008-2012; NASA GLDAS-2 2012, Shiklomanov and Rodda 2004

Fiorke et al. 2012: Matsutomi et al. 2009

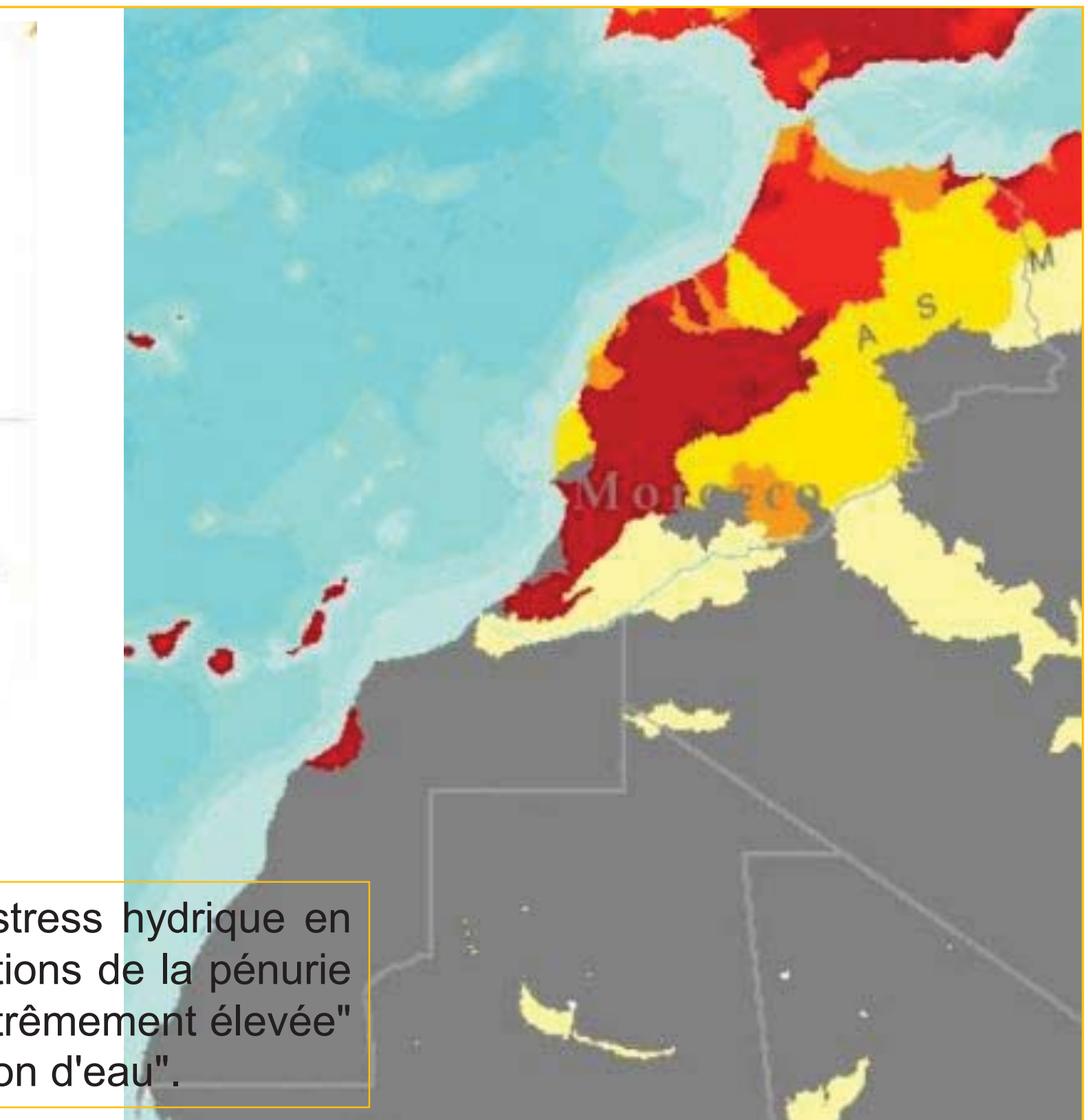

Zones touchées par le stress hydrique en 2010. Légende : graduations de la pénurie de l'eau de "faible" à "extrêmement élevée" et "aride et faible utilisation d'eau". 


\section{RARÉFACTION DES RESSOURCES EN EAU}

La mesure du stress et de la pénurie en eau la plus connue est définie par M. Falkenmark (1986) reprise par F. Lasserre et L. Descroix (2002).

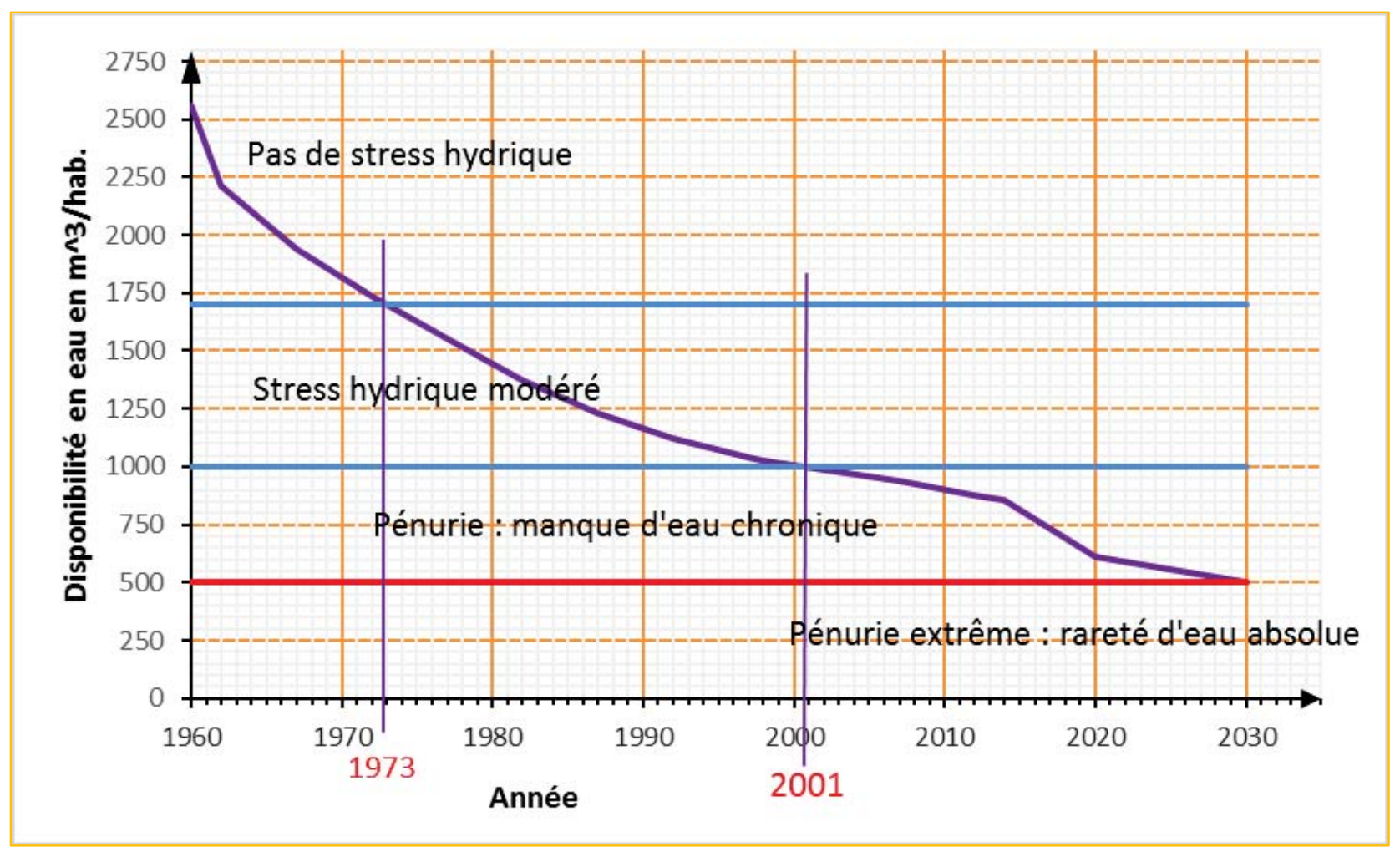

Cette classification permet de placer le Maroc dans une situation hydrique de manque d'eau chronique qui a perduré 19 ans. Cette quantité pourra chuter à $500 \mathrm{~m}^{3} / \mathrm{hab}$./an vers 2030 , ce qui menacera le pays d'une rareté d'eau absolue. 


\section{SUREXPLOITATION DES RE EAU SOUTERRAINE PAR BASSIN}

\begin{tabular}{|l|c|c|c|c|}
\hline \multicolumn{1}{|c|}{ Bassins } & $\begin{array}{c}\text { Potentiel des } \\
\text { ressources en } \\
\text { eau souterraine } \\
\text { en Millions de m }\end{array}$ & $\begin{array}{c}\text { Ressource en eau } \\
\text { souterraines } \\
\text { exploitables en } \mathrm{Mm}^{3} \\
\text { (gestion durable) }\end{array}$ & $\begin{array}{c}\text { Potentiel } \\
\text { mobilisé } \\
\text { en } \mathrm{Mm}^{3}\end{array}$ & $\begin{array}{c}\text { Volume } \\
\text { surexploité } \\
\text { en } \mathrm{Mm}^{3}\end{array}$ \\
\hline $\begin{array}{l}\text { Loukkos, Tangérois et côtiers } \\
\text { méditerranées }\end{array}$ & 189 & 110 & 110 & 0 \\
\hline Moulouya, Figuig - Kert - Isly - Kiss & 512 & 407 & 462 & -55 \\
\hline Sebou & 1301 & 1041 & 1143 & -102 \\
\hline Bouregreg et la Chaouïa & 116 & 103 & 146 & -43 \\
\hline Oum Er Rbiâa et El Jadida Safi & 406 & 335 & 457 & -122 \\
\hline Tensift et Ksob Igouzoulen & 522 & 518 & 753 & -235 \\
\hline Souss Massa et Tiznit Ifni & 371 & 369 & 646 & -277 \\
\hline Drâa & 371 & 296 & 323 & -27 \\
\hline Ziz Rhéris Guir Bouâanane Maider & 301 & 208 & 208 & -0 \\
\hline Bassins Sahariens & 17 & 17 & 18 & -1 \\
\hline Total (Millions de m ${ }^{3}$ /an) & 4106 & 3404 & 4266 & -862 \\
\hline
\end{tabular}




\section{SUREXPLOITATION DES NAPPES}
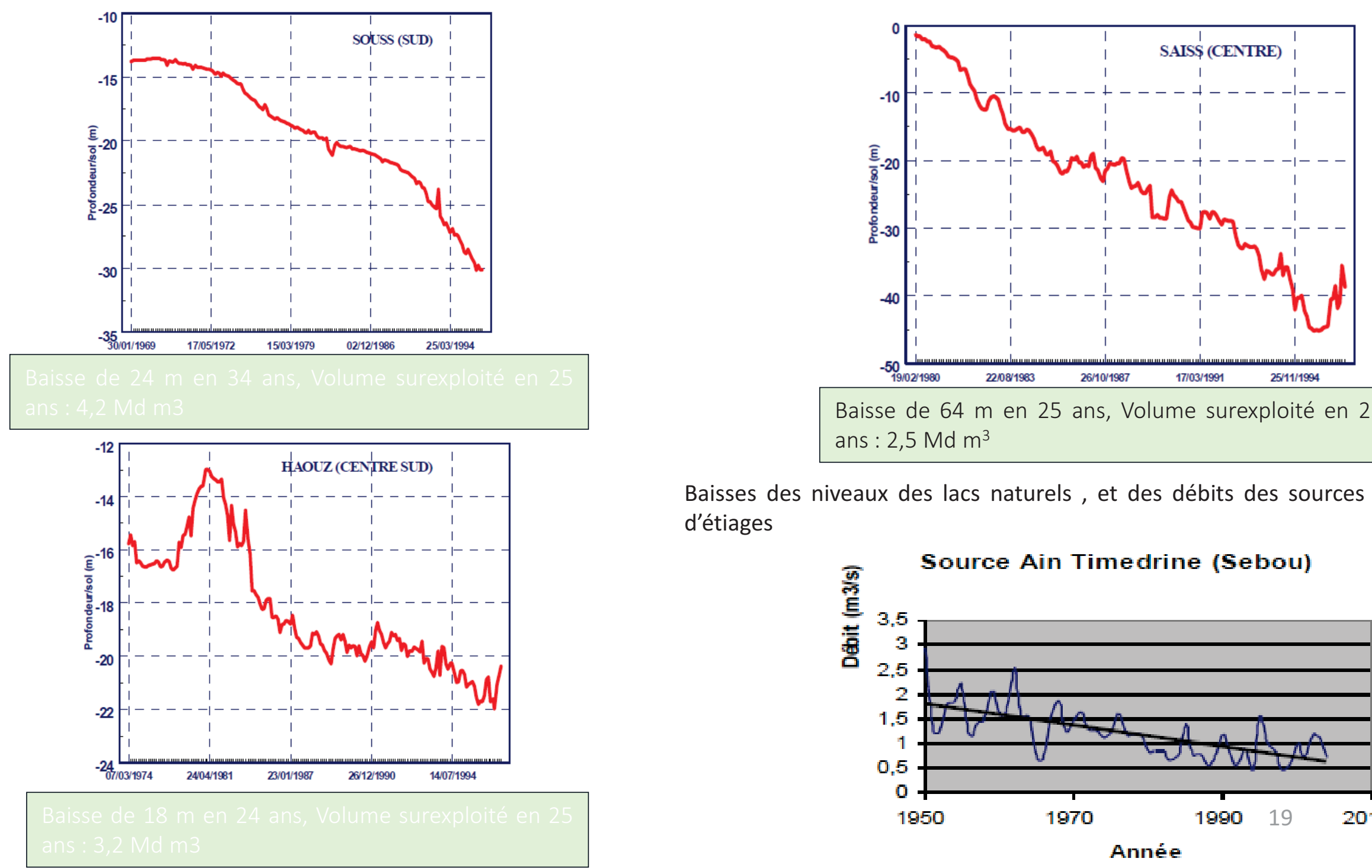

Baisse de $64 \mathrm{~m}$ en 25 ans, Volume surexploité en 25 ans : $2,5 \mathrm{Md} \mathrm{m}^{3}$

Baisses des niveaux des lacs naturels, et des débits des sources et d'étiages

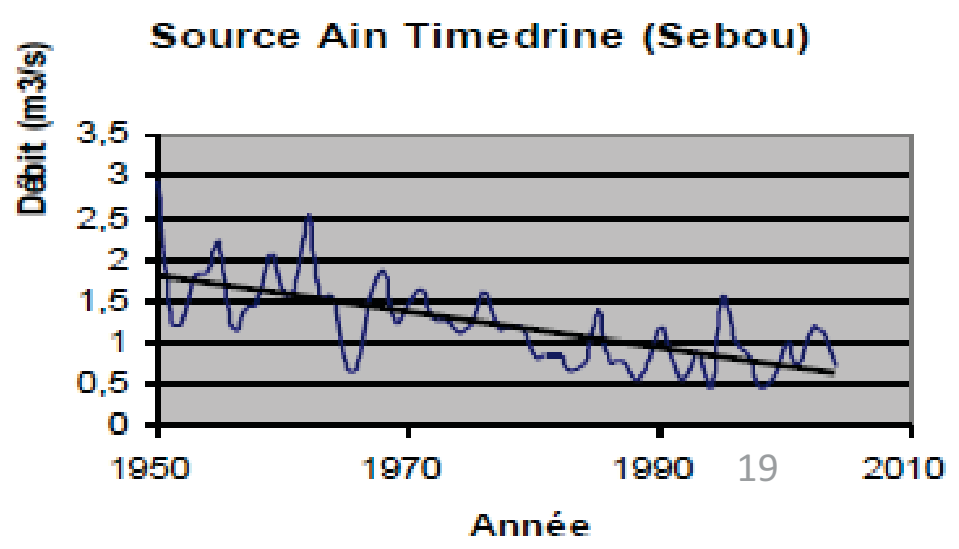


Baisse alarmante dans la plupart des nappes, quasi- épuisement de certaines nappes et intrusion des eaux marines au niveau des nappes d'eau souterraine côtières.

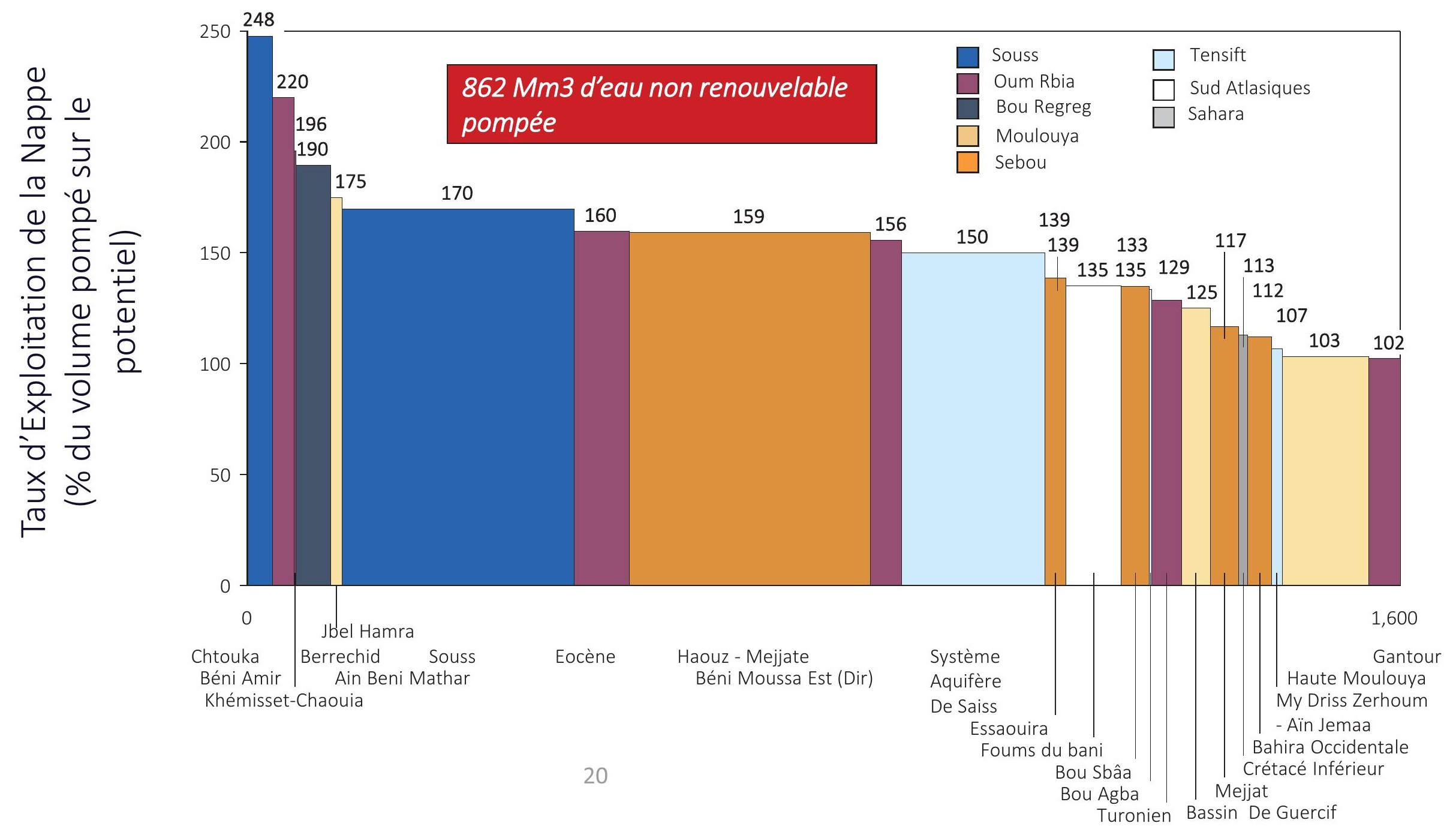




\section{LES SORTIES DU SYSTÈME}

Volumes d'eau bleue perdus vers l'océan, la mer et à l'extérieur des frontières SE. durant l'année 20092010 est de 37 milliards de $\mathrm{m}^{3}$ qui est en baisse continue avec la construction des barrages. Le tout à barrage !

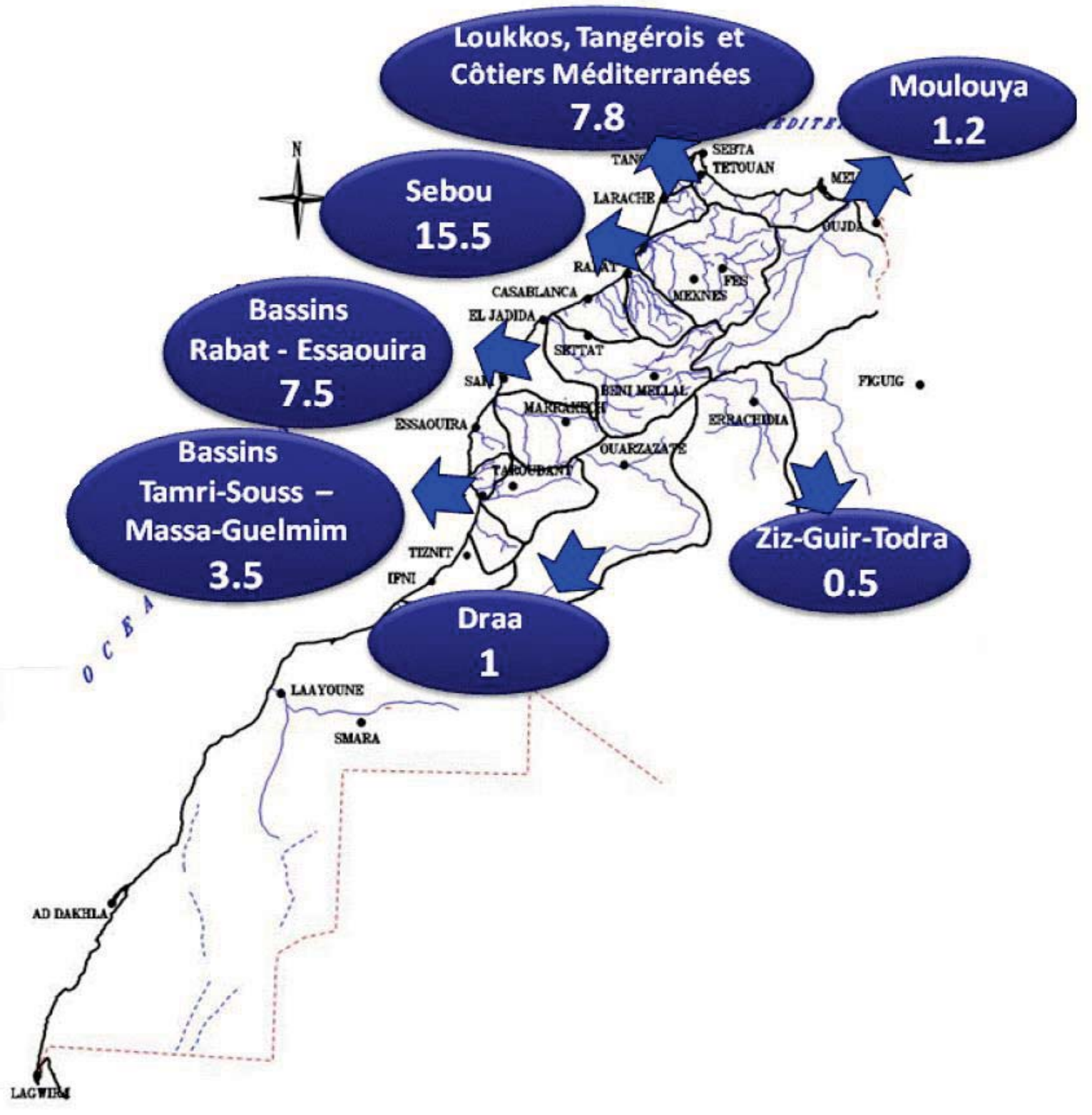




\begin{tabular}{|c|c|c|c|}
\hline Précipitations & & 140 & \\
\hline Évapotranspirations & & & 118 \\
\hline $\begin{array}{c}\text { Totales des ressources } \\
\text { en eaux renouvelables }\end{array}$ & & 22 & \\
\hline $\begin{array}{c}\text { Ressources en eaux } \\
\text { mobilisables }\end{array}$ & de surface & & souterraine \\
\hline $\begin{array}{c}\text { Ressources en eaux } \\
\text { mobilisées }\end{array}$ & 18 & & 3,4 \\
\hline
\end{tabular}

Chiffres en milliards de $\mathrm{m}^{3} / \mathrm{an}$

Potentiel des ressources en eau du Maroc

La part du volume des réserves, physiquement mobilisable, est en moyenne de $50 \%$ pour les nappes libres importantes et de $10 \%$ pour les nappes captives.

Ces chiffres sont seulement les ressources en eau renouvelable. Le volume surexploité est de l'ordre de 0,86 milliards de $\mathrm{m}^{3}$ /an 


\section{CONFRONTATION DEMANDES/RESSOURCES}

La confrontation entre la demande et l'offre montre qu'il y a un déséquilibre hydrique en 2020. Donc une perception de la crise de l'eau causée par la croissance de la demande. Les solutions proposées créent des grands chantiers pour les entreprises internationales qui créent des emplois au détriment de l'augmentation de la pauvreté.

\begin{tabular}{|c|c|c|c|c|c|c|c|c|c|}
\hline \multicolumn{5}{|c|}{1990} & \multicolumn{5}{|c|}{2020} \\
\hline \multicolumn{2}{|c|}{$\begin{array}{c}\text { Ressources en } \\
\text { eau } \\
\text { milliards de } \\
\mathrm{m}^{3} / \mathrm{an}\end{array}$} & \multicolumn{3}{|c|}{$\begin{array}{l}\text { Demandes en eau } \\
\text { milliards de } \mathrm{m}^{3} / a n\end{array}$} & \multicolumn{2}{|c|}{$\begin{array}{c}\text { Ressources en } \\
\text { eau } \\
\text { milliards de } \\
\mathrm{m}^{3} / \mathrm{an}\end{array}$} & \multicolumn{3}{|c|}{$\begin{array}{l}\text { Demandes en eau } \\
\text { milliards de } \mathrm{m}^{3} / \mathrm{an}\end{array}$} \\
\hline $\begin{array}{l}\text { RE } \\
\text { surf. }\end{array}$ & $\begin{array}{l}\mathrm{RE} \\
\text { sout. }\end{array}$ & $\begin{array}{c}\text { Irrigatio } \\
\mathrm{n}\end{array}$ & AEP & $\begin{array}{c}\text { Autre } \\
s\end{array}$ & RE surf. & $\begin{array}{c}\mathrm{RE} \\
\text { sout. }\end{array}$ & $\begin{array}{c}\text { Irrigatio } \\
\mathrm{n}\end{array}$ & AEP & $\begin{array}{l}\text { Autre } \\
\mathrm{s}\end{array}$ \\
\hline 8,5 & 2,5 & 8,7 & 1,4 & 0,8 & 14 & 2,8 & 13,5 & 3,6 & 0,5 \\
\hline \multicolumn{2}{|c|}{11} & \multicolumn{3}{|c|}{10,9} & \multicolumn{2}{|c|}{16,8} & \multicolumn{3}{|c|}{17,6} \\
\hline \multicolumn{5}{|c|}{ Demande $\approx$ Ressources } & \multicolumn{5}{|c|}{ Demande $>$ Ressources } \\
\hline
\end{tabular}




\section{QUALITÉ DES RESSOURCES EN EAU}

L'état actuel de la qualité des ressources en eau au Maroc est sujet à une pression sans cesse croissante, due à l'accroissement démographique, l'urbanisation, l'essor industriel et le développement de l'agriculture intensive. Pour la plupart des centres urbains et des industries, les eaux usées sont rejetées sans traitement préalable dans le milieu récepteur. Les eaux de surface sont particulièrement touchées au niveau des zones urbaines. Les eaux usées domestiques sont le plus grand facteur de dégradation de la qualité des eaux de surface, suivies par les rejets industriels, les déchets solides et la pollution d'origine agricole. 
Les résultats de la qualité globale des ressources en eau de surface (2011-2012) montrent que :

- les cours d'eau ont été globalement de qualité bonne à moyenne,

- les points situés en aval des rejets urbains, $4 \%$ ont présenté une eau de qualité excellente, $50 \%$ une eau de bonne qualité, $17 \%$ des stations une moyenne qualité et $29 \%$ des stations étaient de qualité mauvaise à très mauvaise.
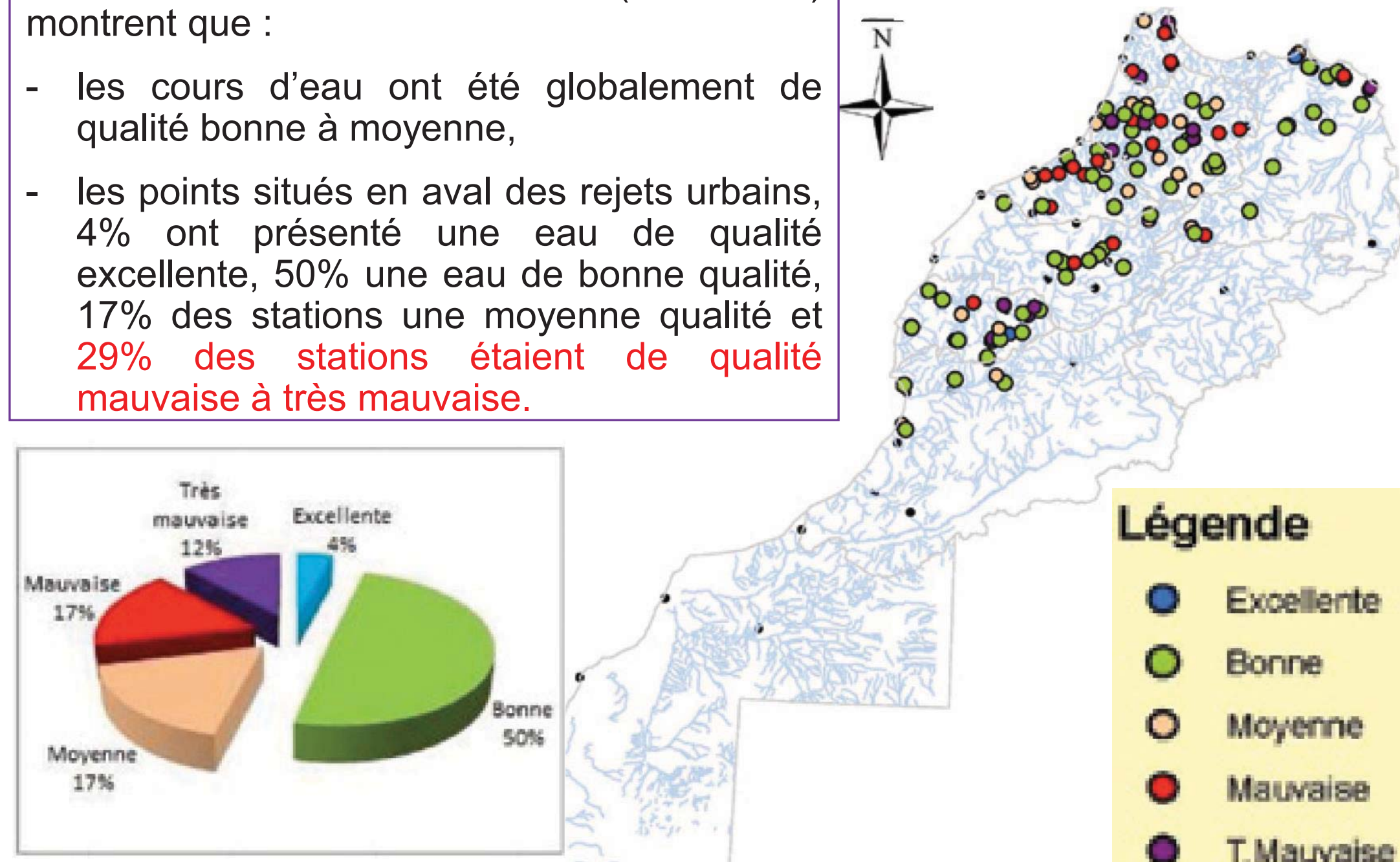

Fig. Qualité des principaux cours d'eaux à l'échelle nationale 


\section{QUALITÉ DES RESSOURCES EN EAU}

- En général, $53 \%$ des stations d'eaux souterraines ont été de qualité excellente à moyenne contre $47 \%$ de qualité dégradée.

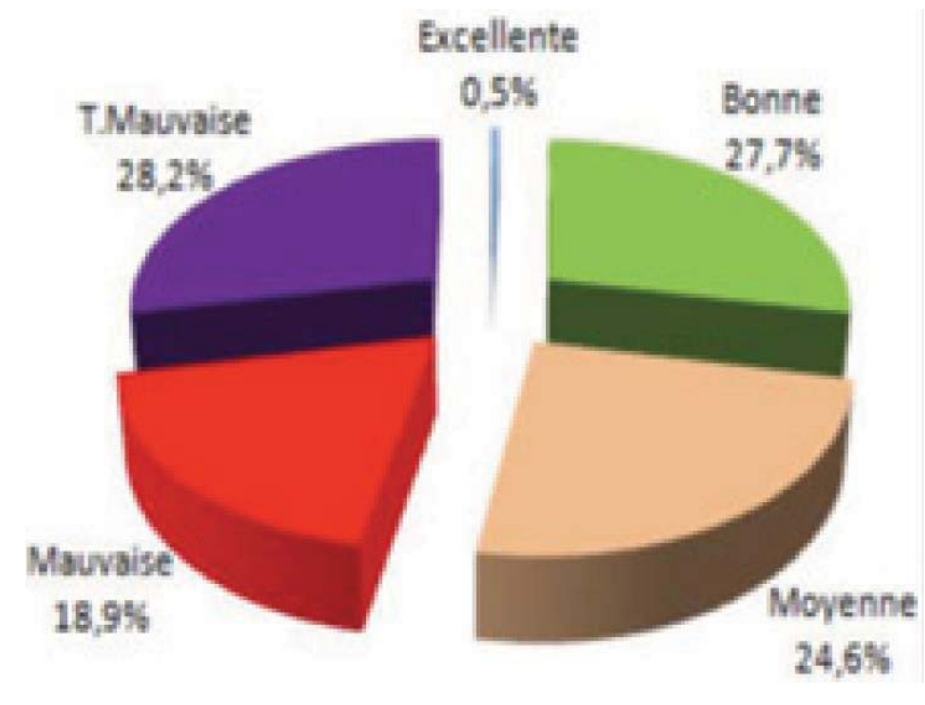

Fig. Qualité des principales nappes d'eaux souterraines à l'échelle nationale

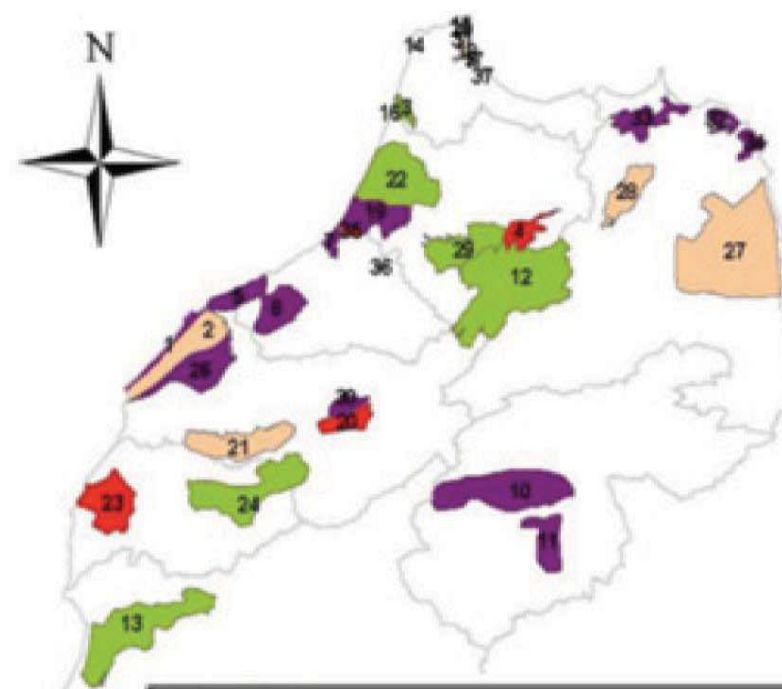

Nom de Nappe

1 Frange cobtiere du she

2 sakel drabda Doukals

3 Pisine alluwiale Ouled Ougbane

4 du couloir fes-Taza

5 Chaowla

65 shara

7 Temara

3 Berrechly

9 AIU

10 Bassin dritrachidia

11 taflialet

12 du Moyen Atlas

13 Nuppes du Souss Chitovia

14 Chart el abab

Is rinidek

16 Rmedl

17 tims

18 martil
19 Masmora

20 Beni Mousu

$\boldsymbol{z}$ buhin

$\boldsymbol{n}$ Gharb

23 Meshala rourimate

24 Haour

7 Nevo

No Doukaly

$\boldsymbol{z}$ Ain Beed Muthar

as Guerdif

$\boldsymbol{x}$ couloli Fes Mthes

10 Betai Amir

II Smir

12. Trifa

II Kert Gureb bou Avec:

34 Angad

Ms Tanoubart

37 Oued Lave 


\section{INONDATIONS}

Le phénomène des inondations au Maroc a commencé à être ressenti plus fortement depuis les années 1990, en raison d'une part, du développement urbain, industriel et touristique entraînant une occupation croissante des zones vulnérables et d'autre part, de l'aggravation des phénomènes extrêmes suite aux changements climatiques.

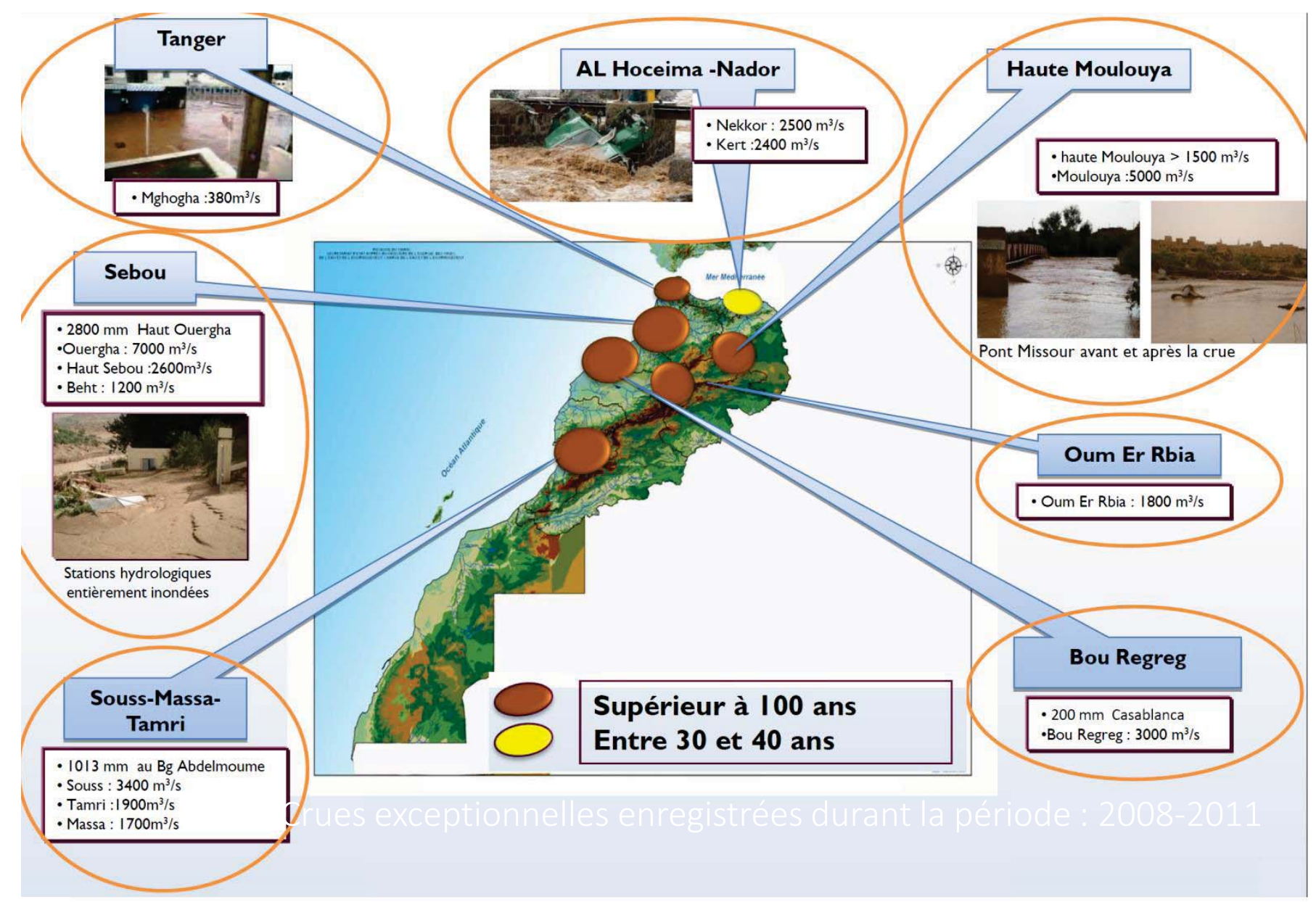

Impact : pertes économiques importantes avec des dégâts matériels et humains 


\section{MANIFESTATIONS DE SOIF}

Dans l'ensemble du Royaume, le Plan Maroc Vert, a accordé des subventions et des autorisations aux creusements de puits et forages pour les besoins de l'agriculture avec un rythme de deux, quatre, voire cinq puits sur 100 hectares. Ce qui va augmenter la baisse des nappes.

Les habitants de la commune Talsint de 16000 habitants en 2018, depuis plusieurs mois, élèvent leur voix à cause de la coupure des robinets dans les foyers pendant deux, trois voire

cinq heures» accès régulier à l'eau potable !

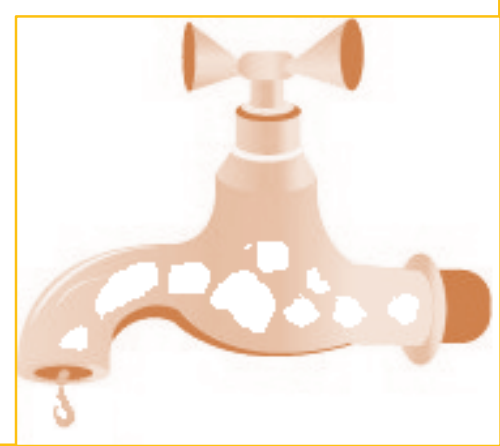




\section{MANIFESTATIONS DE SOIF}

- Zagora a connu au début de l'été 2017 , des " manifestations de la soif ", après plusieurs semaines d'accès à l'eau non régulier, qui plus est, imbuvable. Cette commune est connue par la culture de la pastèque connue par une excellente réputation sur le marché marocain !

- Des coupures plus ou moins régulières d'eau potable ont été enregistrées en 2019 dans plusieurs localités du pays : Ouezzane, Oulad Berhil (Taroudant), Oulad Khalouf (Ghalaât Sraghna), Sidi Bennour (Al Jadida), Moulay Bouazza (Khénifra), Oujda et même Casablanca. Dans la majeure partie de ces localités (Oulad Berhil, Oulad Khallouf, Moulay Bouazza...), les populations ont soit organisé des sit-in de protestation sur place, soit marcher vers les sièges des administrations territoriales (wilaya, préfecture...) dont ils relèvent pour exiger que cessent ces coupures intempestives d'eau potable en pleine période de fortes chaleurs estivales.
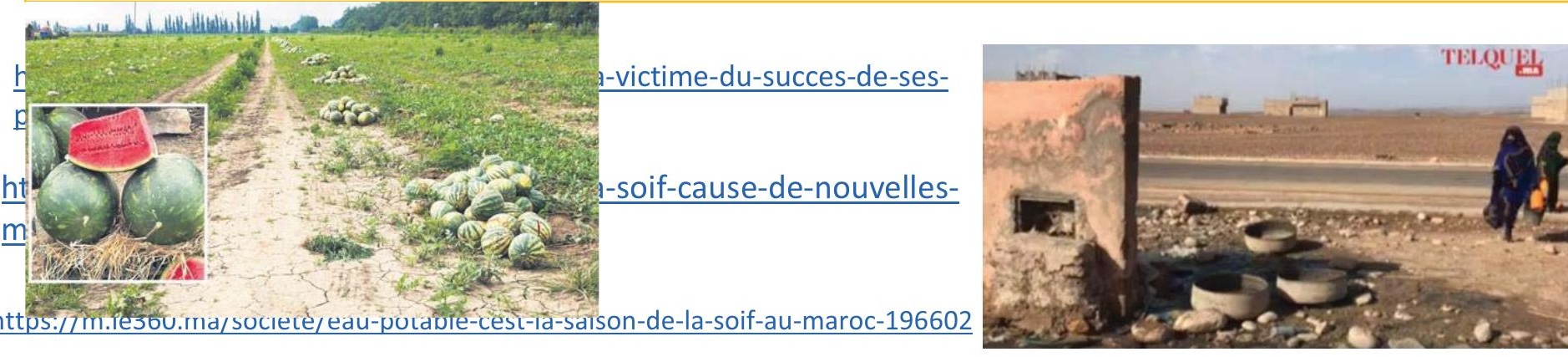


\section{INFRASTRUCTURE HYDRAULIQUE DU MAROC}

- Le pays dispose actuellement de :

- plus de 145 grands barrages,

- +de 13 ouvrages hydrauliques de transferts des eaux interbassins,

- plus d'une centaine de petits barrages et de lacs collinaires,

- d'autres projets en cours de réalisation comportent 38 autres barrages en cours de réalisation répartis en :

- 20 grands barrages 2020-2027,

- 26 petits barrages,

- 255 barrages collinaires

- 14 aménagements de protection contre les inondations.

- En 2018, les retenues des principaux barrages nationaux tous usages confondus ont atteint plus de :

- 9,44 milliards de $\mathrm{m}^{3}$ en 2018,

- 7,2 milliards de $\mathrm{m}^{3}$ en 2019 ;

- 7,52 milliards de $\mathrm{m}^{3}$ en 2020. 


\section{MOBILISATION DES RESSOURCES EN EAU}

- Cadence de construction des grands barrages :

- 1 barrage/an jusqu'à l'an 2000,

- 2 barrages/an jusqu'à 2010,

- 3 barrages/an au-delà de 2010.

- 2 à 3 grands barrages/an à partir de 2010.

- 20 barrages entre 2020-2027

139 grands barrages cumulent une capacité de près de 17,60 milliards de $\mathrm{m}^{3}$ (2014),

80 grands barrages en 1992 dont la capacité totale était de 9,86 milliards de $\mathrm{m}^{3}$ ). Ils servent à l'irrigation, l'AEP, la protection contre les inondations, la recharge des nappes, la protection contre l'envasement, la production d'énergie propre et l'abreuvement du cheptel.

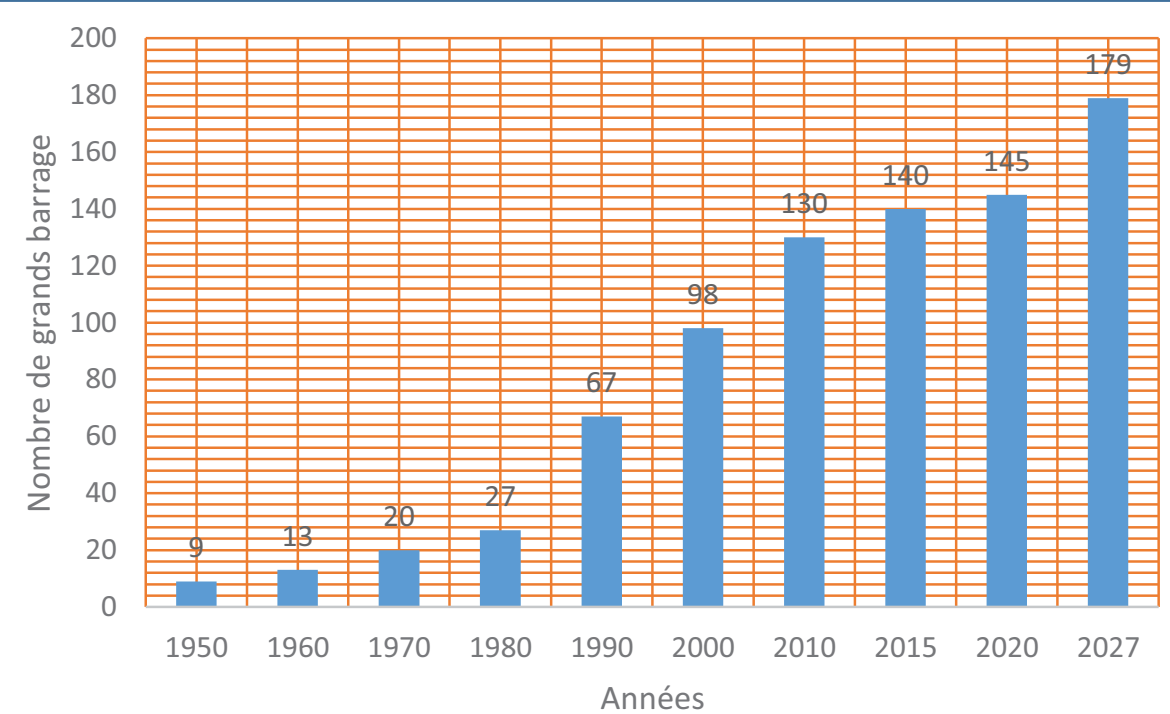




\section{OUVRAGES DE TRANSFERTS N-S, DESSALEMENT}

Unités de dessalement de l'ONEP

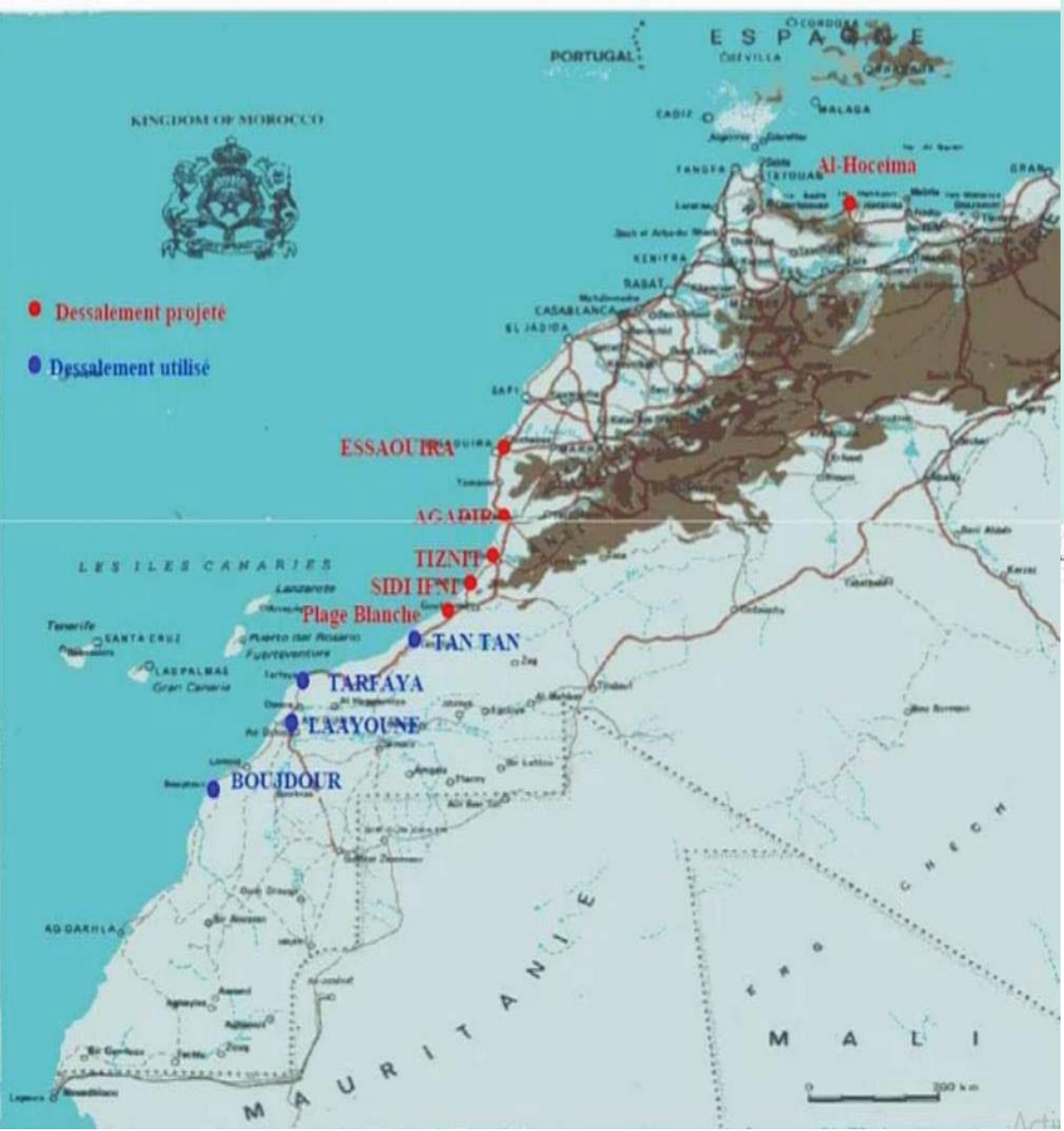

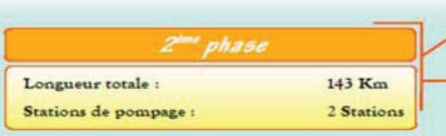
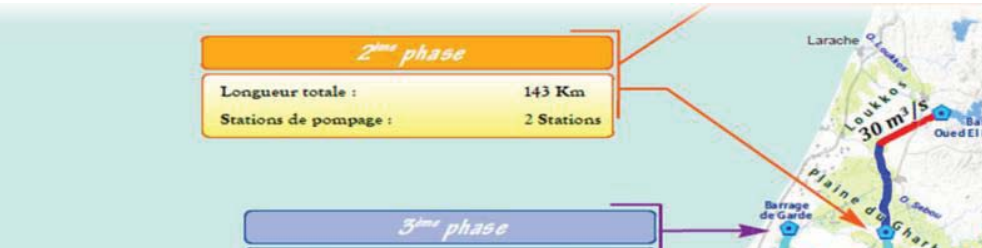

GH Doukkal

Couchane

Berrechid

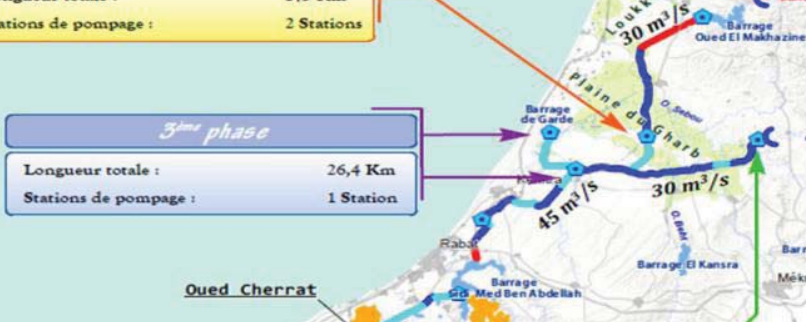
wimend

\section{Nappe de Berrechid}

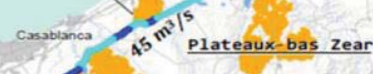

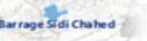
weknes. 


\section{ÉNERGIES PROPRES}

La production électrique renouvelable nationale (éolienne, hydroélectrique et solaire) est passée de 9\% en 2012 à 15\% en 2017.

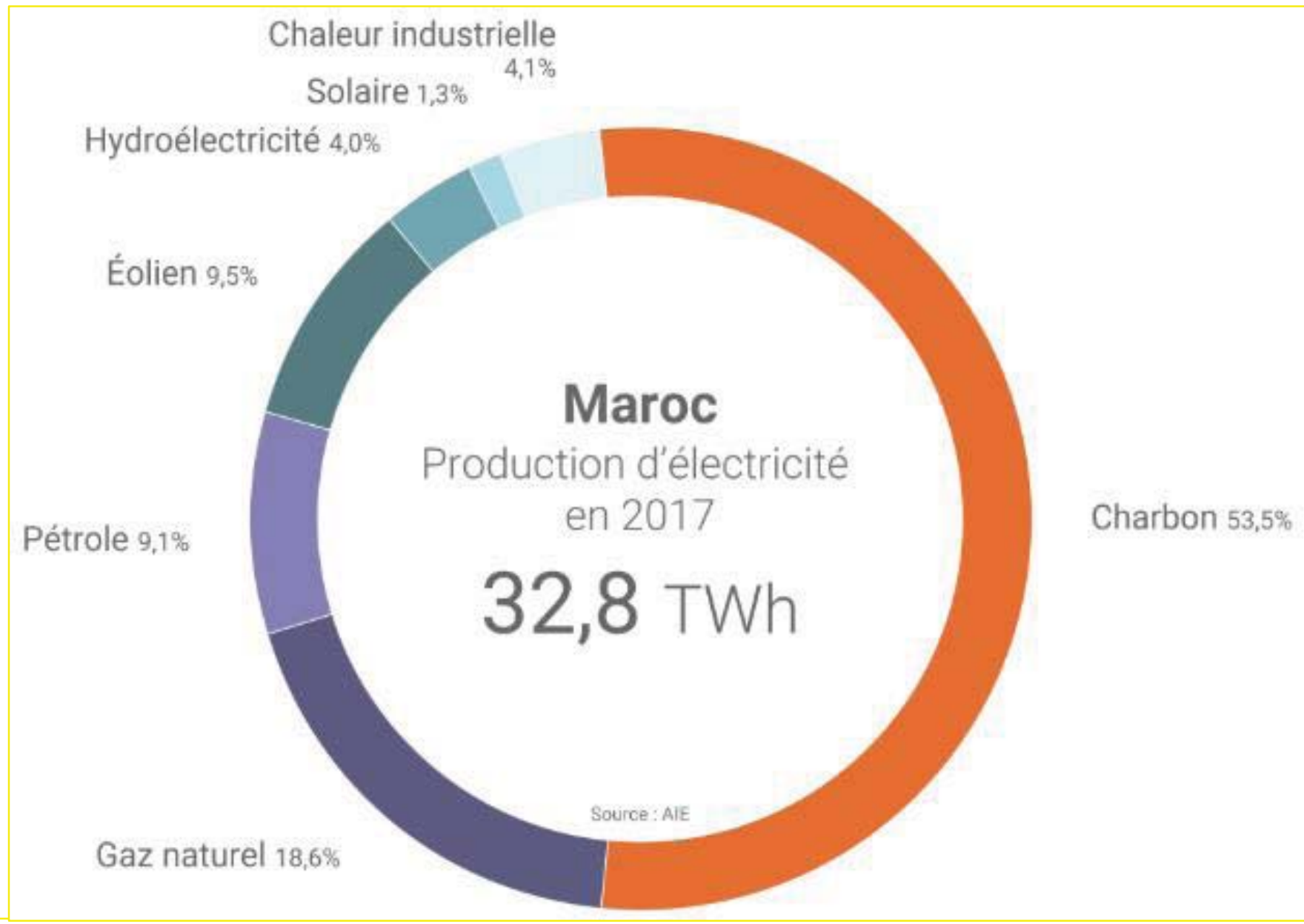

Fig. En 2017, la production électrique du Maroc a reposé à plus de $80 \%$ sur les énergies fossiles. (C) Connaissance des Énergies, d'après AIE)

https://www.connaissancedesenergies.org/la-situation-energetique-du-marocdecryptee-par-laie-190516

https://atlasocio.com/etats/fiches/m/maroc.php 



\section{LOCALISEE}

\begin{tabular}{|c|c|c|c|}
\hline Zone & $\begin{array}{l}\text { Superficie à } \\
\text { équiper (ha) }\end{array}$ & $\begin{array}{l}\text { Coût } \\
\text { (MDH) }\end{array}$ & $\begin{array}{c}\text { Économies d'eau } \\
\text { attendues à terme } \\
\left(\mathrm{Mm}^{3} / \mathrm{an}\right)\end{array}$ \\
\hline 1- ORMVA & 89.500 & 3.050 & 280 \\
\hline - Moulouya & 18.000 & 900 & 60 \\
\hline > Loukkos & 9.500 & 290 & 30 \\
\hline - Tadla & 5.000 & 150 & 20 \\
\hline > Haouz & 20.000 & 600 & 60 \\
\hline - Souss-Massa & 23.000 & 690 & 70 \\
\hline$>$ Gharb (Beht) & 14.000 & 420 & 40 \\
\hline 2. Hors ORMVA & 25.000 & 750 & 80 \\
\hline TOTAL GÉNÉRAL & 114.500 & 3.800 & 360 \\
\hline
\end{tabular}




\section{GESTION DE LA DEMANDE ET VALORISATION DE L'EAU}

Efficience de l'irrigation : Reconversion à l'irrigation localisée : Economie de 2,5 milliards de $\mathrm{m}^{3} / \mathrm{an}$

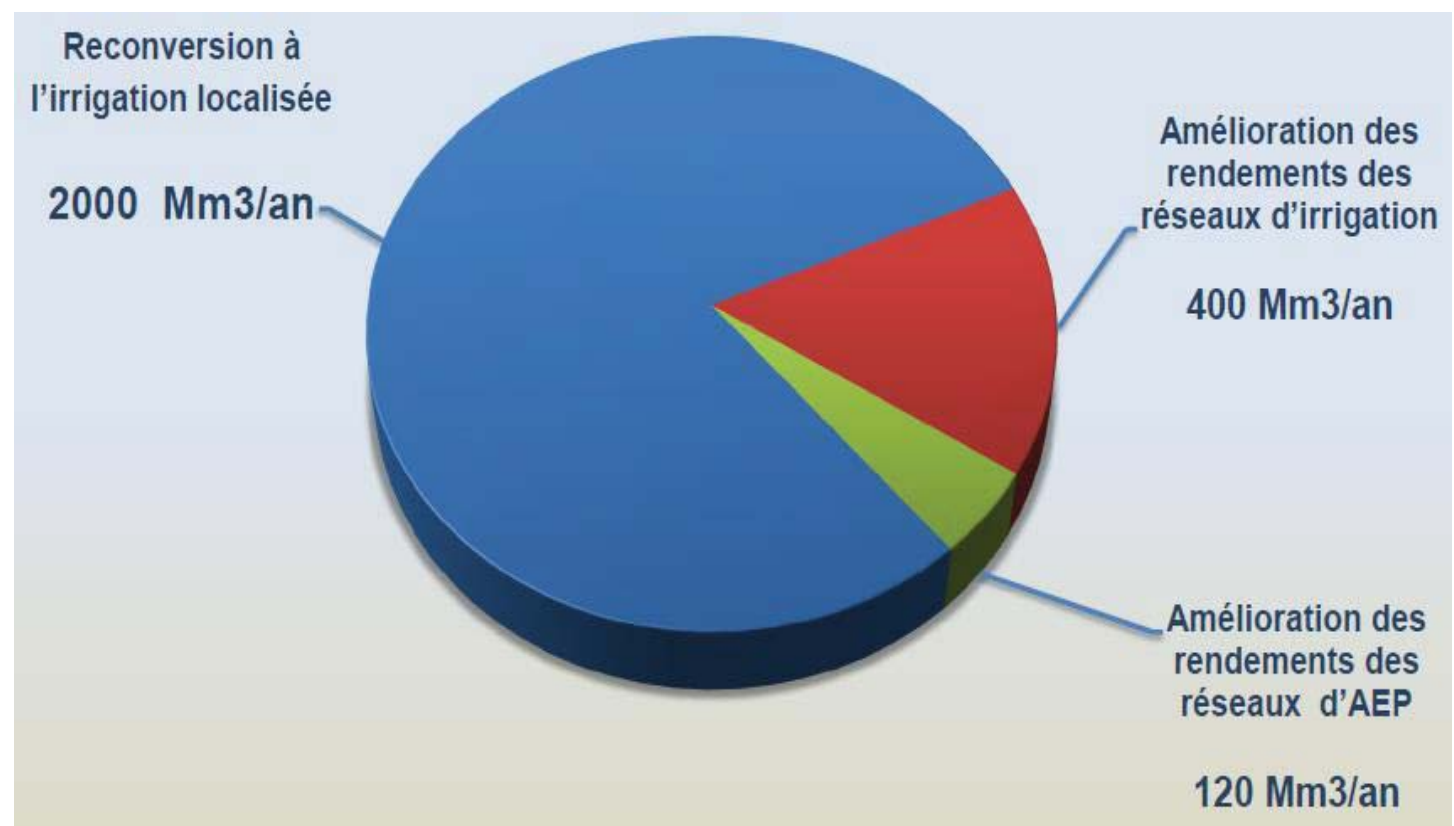

Meilleure adéquation entre aménagement hydraulique et équipement hydro agricole

Programme d'économie d'eau potable, industrielle et touristique 
L'unité spatiale de la GIRE au Maroc est le bassin hydraulique. Chacun de ses bassins a une multitude de problèmes de l'eau (aridité, pollution, ...).

Si l'on croit ces chiffres, le Maroc a fourni beaucoup d'efforts dans la gestion de ses ressources (offre, demande...).

Paradoxalement à l'échelle internationale, on enregistre encore de mauvais scores (parité, pauvreté...)!

La pénurie d'eau est chronique, l'offre est inférieure à la demande, l'exploitation des ressources non renouvelables, la dégradation de la qualité des eaux, sècheresses, inondations, ...

La cause principale de ses problèmes est la mauvaise gestion des ressources en eau. 
Selon I'UNICEF (2016), les 3/4 des ménages qui n'ont pas accès à l'eau de boisson chez eux confient la responsabilité principale de la collecter, l'utilisation et la gestion de l'eau aux femmes et aux filles. Elles sont les principaux promoteurs des activités d'assainissement. Ces travaux ne sont ni rémunérés, ni reconnus.

Dans de nombreuses régions du monde, les femmes et les jeunes filles sont régulièrement victimes de discrimination et d'inégalités dans l'exercice de leurs droits fondamentaux en matière d'accès à l'eau potable et à l'assainissement.

Les menstruations entravent la prise en compte des besoins spécifiques des femmes et des filles en matière d'assainissement, les forçant à utiliser des méthodes sanitaires non hygiéniques et les toilettes seulement après la tombée de la nuit, mettant ainsi leur sécurité en péril. 
Les femmes enceintes sont plus vulnérables aux conséquences des maladies liées à l'eau et à l'assainissement. Les femmes et les filles risquent aussi de subir des violences (physiques, psychiques et sexuelles) lorsqu'elles doivent parcourir de longues distances pour aller chercher de l'eau.

D'une manière générale, les arrangements institutionnels relatifs à la mise en valeur et à la gestion des ressources en eau tiennent rarement compte du rôle primordial des femmes comme utilisatrices d'eau et gardiennes du milieu vivant.

Dans de nombreux pays, les inégalités de genre en matière d'accès à l'eau et à l'assainissement sont importantes et persistantes. Selon l'Indice de développement humain (IDH), les femmes du monde entier disposent d'un IDH inférieur à celui des hommes (une différence allant jusqu'à $20 \%$ en Asie du Sud), ce qui illustre les effets répandus des inégalités dont les femmes sont victimes (PNUD, 2016). 


\section{CORVÉES DE FEMMES}

Dans les zones rurales les femmes:

- assurent l'approvisionnement en eau à l'unité familiale et y consacrent parfois des journées entières. Elles ne s'autorisent donc aucun gaspillage.

- utilisent de l'eau pour la nourriture, la boisson, l'hygiène, l'arrosage des potagers, l'abreuvement des animaux, etc.

- savent localiser les sources locales d'eau de qualité. Elles la recueillent, la stockent et contrôlent son utilisation et sa propreté en la protégeant des pollutions.

- recyclent l'eau et en font une utilisation optimale.

Les femmes expriment une forte conscience des problèmes de rareté de la ressource et par conséquent de la nécessité de l'économiser. Elles sont également très attentives à la question de la qualité de l'eau, qui est jugée globalement bonne. Les femmes apprécient la qualité de l'eau d'irrigation principalement par l'odeur, le goût, l'observation de ses effets sur le matériel d'irrigation et les sols, son degré d'acceptation par les animaux. Elles sont particulièrement conscientes des problèmes de pollution de l'eau par les engrais et pesticides, les rejets industriels et les eaux usées domestiques et sont sérieusement préoccupées par leurs conséquences en matière de santé. 


\section{CORVÉES DES FEMMES}

- Le travail et le foyer occupent $90,7 \%$ de toutes les activités chez la femme marocaine, contre $80,9 \%$ chez l'homme. Les activités des femmes citadines au foyer représentent $62,2 \%$ de toutes ses activités. En milieu rural, son travail au foyer est $54,1 \%$, le reste soit $34,9 \%$ est à l'extérieur de la maison. Le temps consacré pour l'approvisionnement en eau dépasse 2 heures dans $40 \%$ des cas.

- La femme rurale marocaine a maitrisé l'économie circulaire depuis la nuit des temps. Paradoxalement, l'OCP, $n^{\circ} 1$ mondial du phosphate (2018) qui a un besoin en eau de 160 millions de $\mathrm{m}^{3}$ par an environ, n'a lancé son programme d'économie circulaire qu'en 2018 !
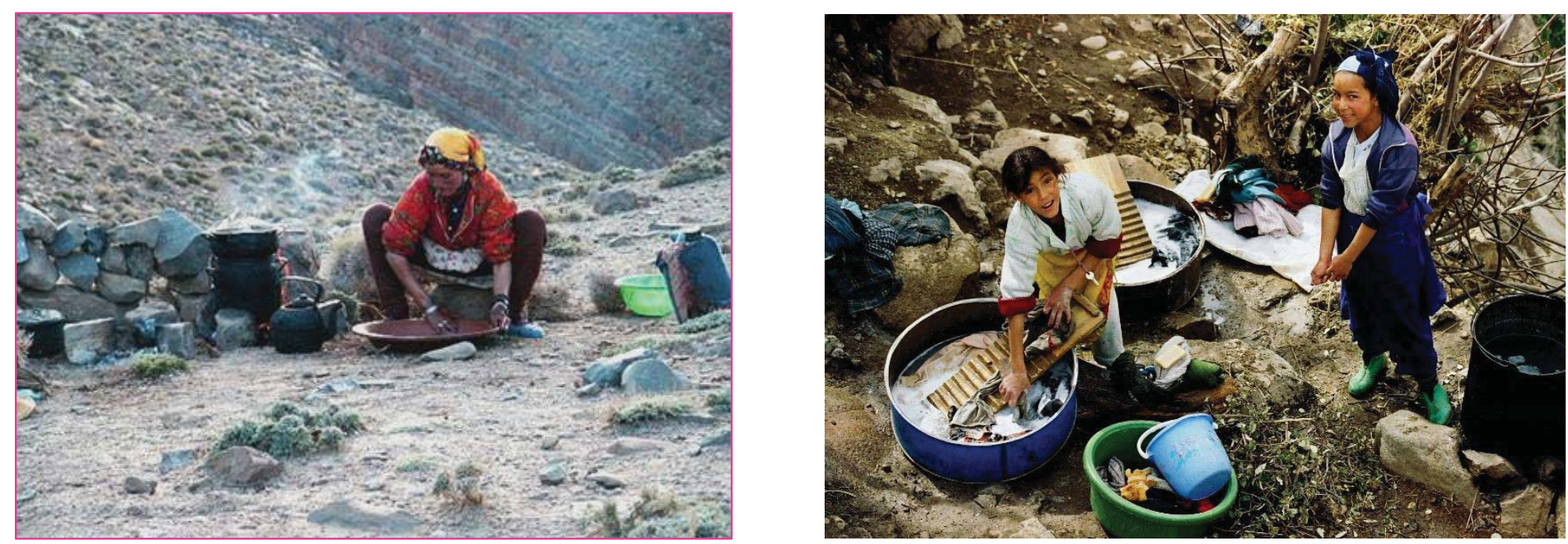


\section{CORVÉES DES FEMMES}

Pour ce qui est de l'approvisionnement en eau, $75 \%$ de femmes rurales déclarent prendre des décisions de leur propre chef. En cas d'absence du mari et d'un fils homme, les femmes assument les responsabilités liées au foyer et à la production. Cependant, le fardeau du travail domestique freine leur activité économique et les profits générés par leurs activités ne leur reviennent que dans $21 \%$ des cas (souvent, il est obtenu par le tuteur ou l'époux). 


\section{CORVÉES DES FEMMES}

L'agriculture reste le secteur premier d'activité des femmes rurales. En $2013,59,5 \%$ des femmes actives travaillent dans le secteur agricole. Néanmoins, ces femmes sont défavorisées sur de nombreux aspects :

- $55 \%$ des femmes rurales sont illettrées et leur accès aux services médicaux est très faible ;

- les femmes ne représentent que $4,5 \%$ du nombre global des exploitants agricoles et ne disposent que de $2,5 \%$ de la surface agricole utile. Les fermes exploitées par des femmes sont des micro-petites exploitations : plus de 7 sur 10 ont une surface agricole utile inférieure à 3 hectares (par rapport à 5 sur 10 pour les hommes);

- les hommes prennent la plupart des décisions, mais les femmes sont communément consultées.

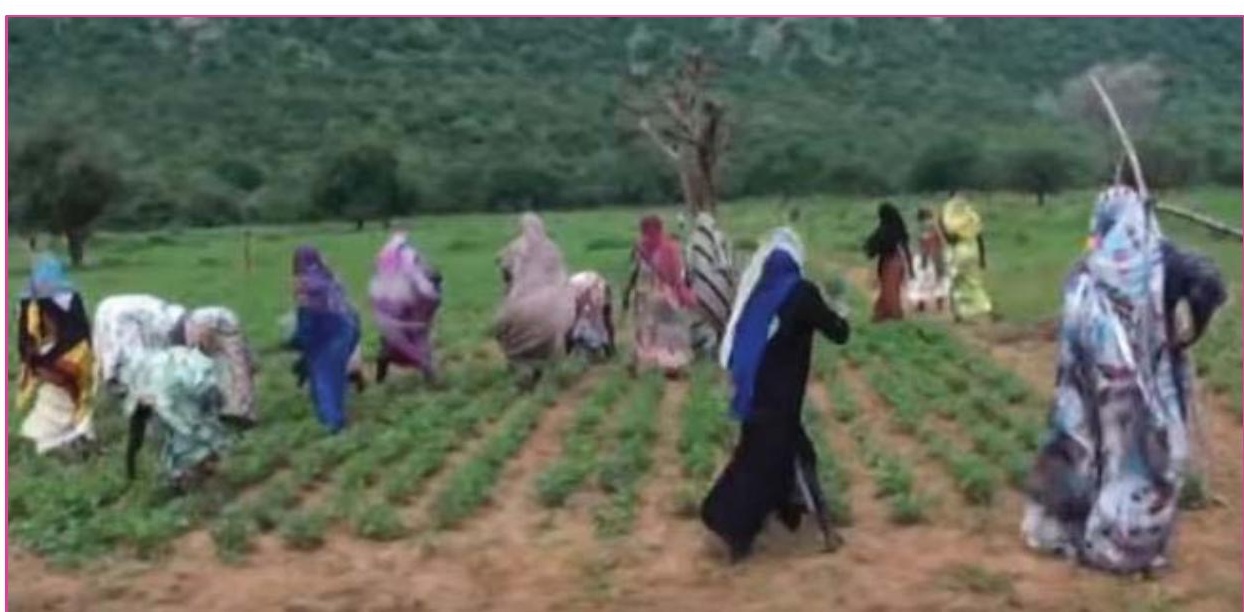




\section{CORVÉES DES FEMMES}

L'activité agricole des femmes reste le plus souvent sous-estimée par les statistiques nationales. Cette faible estimation de la participation des femmes à la production agricole et à sa gestion est étroitement liée aux catégories statistiques utilisées pour évaluer la main d'oeuvre agricole. $\mathrm{Ne}$ sont recensées comme actives que les femmes se déclarant occupées dans l'agriculture. Par ailleurs les femmes sont le plus souvent assimilées à la main d'oeuvre familiale, même si elles jouent un rôle important dans la gestion de l'exploitation.

Il y a un décalage entre ce que les femmes font comme travail et leurs accès à la propriété, aux crédits et aux associations des usagers d'eau. 


\section{CORVÉES DES FEMMES}

\section{Problème d'accès à l'eau potable et à l'assainissement}

Les ruraux migrent vers les villes pour une vie meilleure. Ils s'installent dans le zones périurbaines. Le statut informel ne leur permet pas l'accès à des services tels que l'eau, l'assainissement. Ces populations connaissent des retards d'accès à l'eau et à l'assainissement.

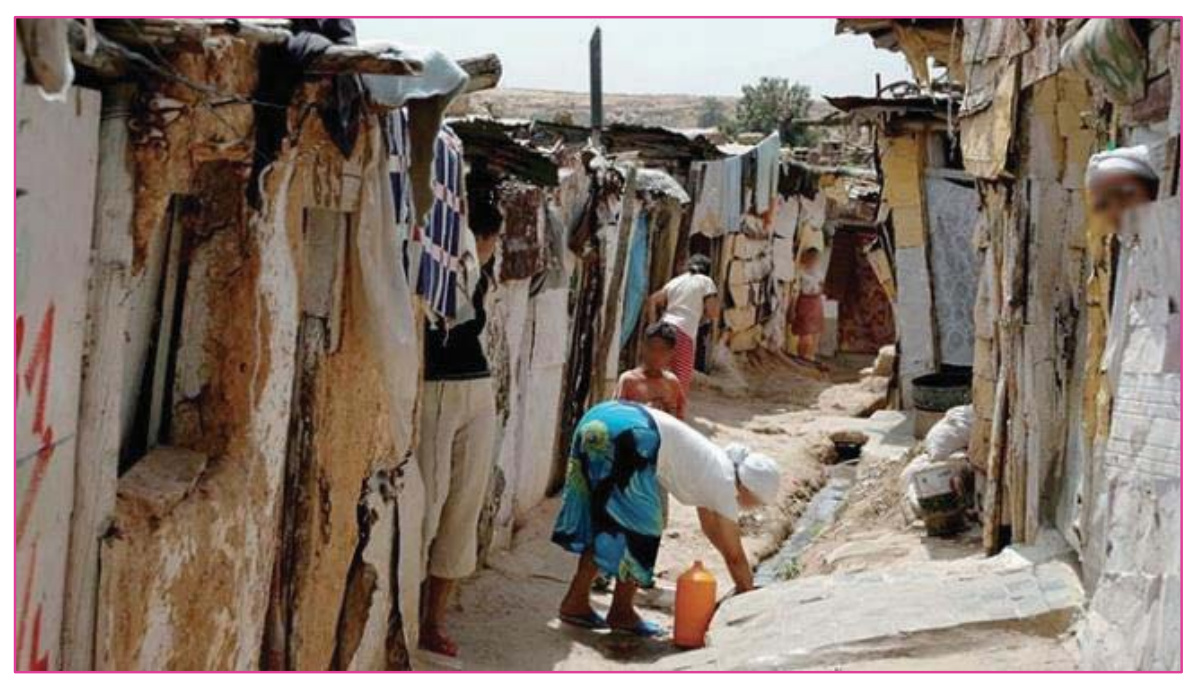




\section{CORVÉES DES FEMMES}

Ce sont les femmes qui évacuent les eaux usées dans la nature. Au fil de l'eau, les femmes rurales en amont polluent l'eau pour ses voisines en aval. Avec l'accroissement de l'accès à l'eau, cette pratique a régressé.

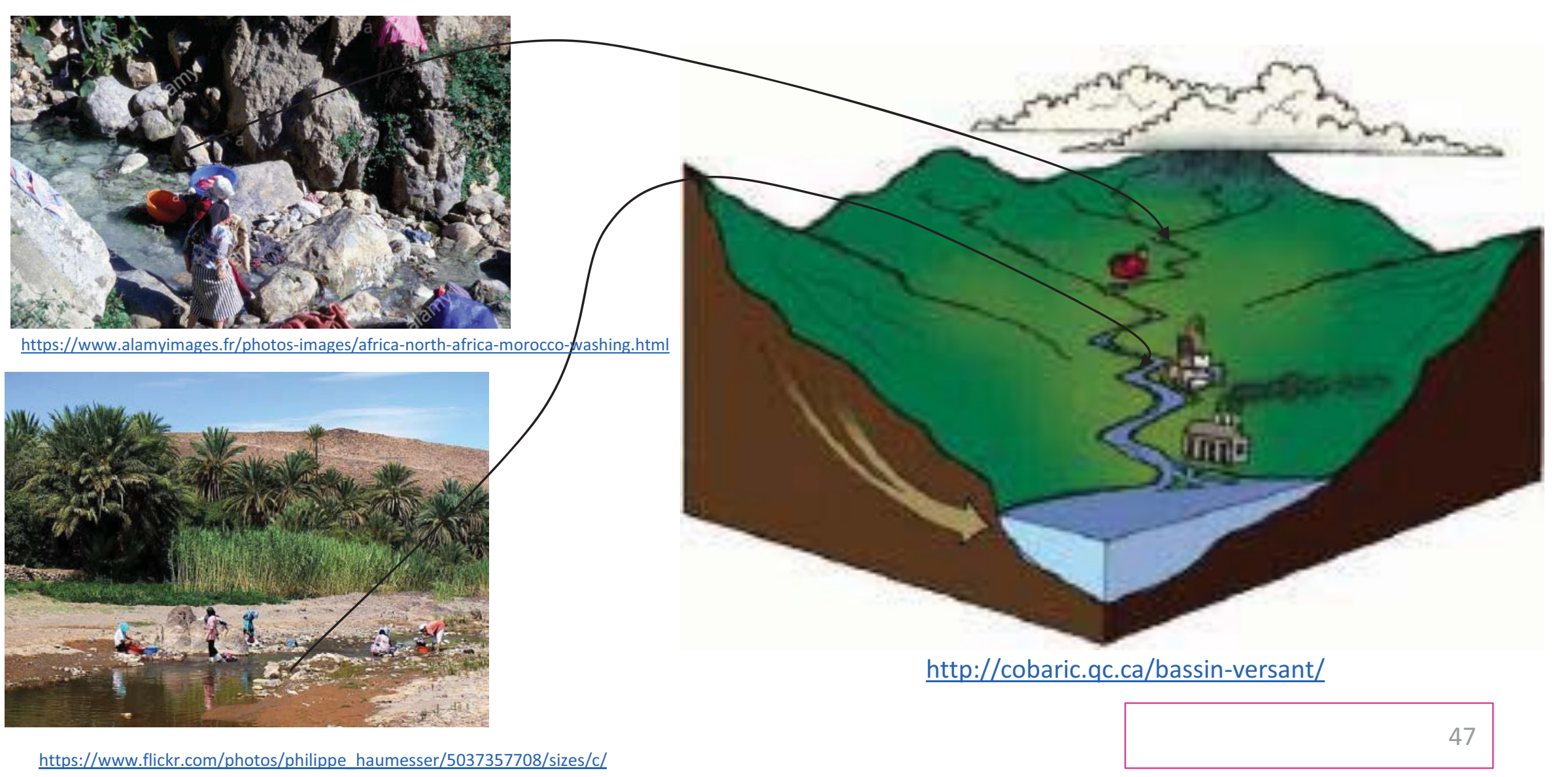




\section{ANALPHABÉTISME}

D'après le HCP, 8,5\% de la population âgée de 25 ans et plus, ont atteint le niveau supérieur des études en 2016 , contre $7,9 \%$ en 2015. Cette proportion est plus élevée chez les hommes $(10,6 \%$ contre $9,8 \%$ en 2015$)$ que chez les femmes $(6,6 \%$ contre $6,2 \%$ en 2015).

Pour ceux qui ont atteint le niveau secondaire (collégial ou qualifiant ou post secondaire) ils représentent $24,0 \%$ contre $23,8 \%$ en 2015 . Le reste de la population, soit $44,0 \%$ n'a jamais fréquenté un établissement scolaire ou est illettré $(57,9 \%$ pour les femmes et $28,2 \%$ pour les hommes).

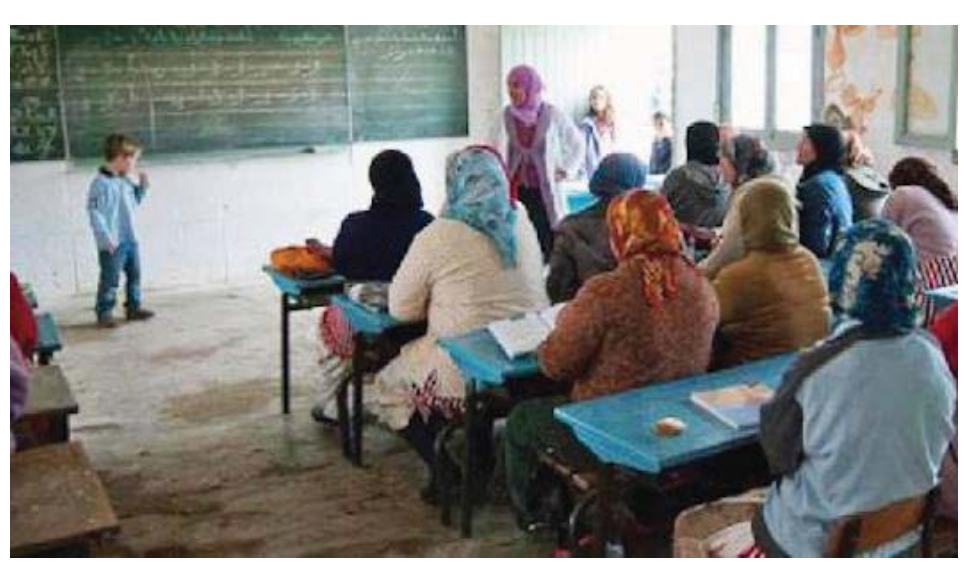

https://www.medias24.com/MAROC/SOCIETE/188970-Enseignement-85-des-Marocains-ages-de-25-ans-et-plusont-atteint-le-niveau-superieur.html 


\section{ANALPHABÉTISME}

L'analyse des données du dernier recensement de 2014 relève une baisse du taux d'analphabétisme de la population âgée de 10 ans et plus. Par milieu de résidence, cette baisse est plus prononcée chez les femmes en milieu rural, soit de $74,5 \%$ en 2004 à $60,1 \%$ en 2014 , qu'en milieu urbain soit seulement de $39,5 \%$ à $31,0 \%$.

L'abandon scolaire est un acte expliqué par plusieurs causes notamment le déplacement des élèves d'une commune à l'autre ou même l'envie de ne plus continuer les études. Les taux d'abandon varient selon le niveau et le sexe. En 2015/16 et au primaire, ces taux sont plus élevés chez les filles $(2,5 \%)$, la proportion des garçons quittant l'école est de $1,5 \%$. On relève $12,7 \%$ au secondaire collégial comme taux d'abandon chez les garçons contre $8,5 \%$ enregistré chez les filles, et au secondaire qualifiant $12,7 \%$ et $10,3 \%$ enregistrés respectivement chez les garçons et les filles.

L'absence d'assainissement et d'installations sanitaires en matière d'hygiène menstruelle dans les écoles et sur les lieux de travail contribue à des taux élevés d'absentéisme féminin, ce qui entraîne une discrimination accrue à l'égard des femmes sur le marché du travail. 


\section{PAUVRETÉ DE LA FEMME RURALE}

D'après le rapport de la SNDD les femmes constituent encore une catégorie sociale pauvre et vulnérable surtout dans le rural. La femme rurale d'une manière particulière, y est généralement plus pauvre que l'homme et possède un accès limité aux ressources et aux services.

La pauvreté de la femme est multidimensionnelle et se mesure par rapport à ses capacités à faire face aux différents problèmes de santé, d'éducation, de mobilité, d'accès aux ressources et de transformer les opportunités offertes en de réelles potentialités à exploiter.

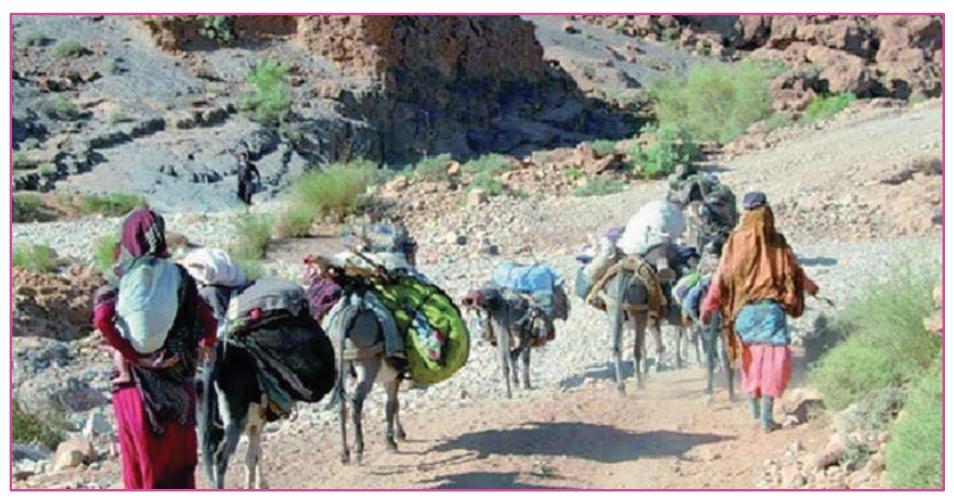




\section{INÉGALITÉ DE GENRE}

L'index des disparités par genre montre un fort écart entre les hommes et les femmes. Le Maroc se classe dans les cinq derniers pays ( $125^{\mathrm{e}}$ sur 130 pays) en 2008. Au Maroc des enquêtes sur 116 femmes dans quatre sites (AQUASTAT, 2014) ressort le constat général qu'il y a un déséquilibre entre les femmes et les hommes en ce qui concerne l'accès et le contrôle de l'eau agricole, la visibilité de leur travail dans l'agriculture, l'accès à la formation et l'opportunité de participer aux structures politiques et les organisations de gestion de l'eau.

Le rapport national du Maroc souligne que le droit coutumier réglementant les terres collectives exclut les femmes de l'accès à la propriété.

Les programmes de développement ne font pas bénéficier tout le monde d'une manière égalitaire, notamment au sein de la famille.

En zone montagneuse, le taux des femmes chefs de ménages ne dépasse pas $10 \%$ et reste inférieur au taux national de $16,1 \%$ en 2011. 


\section{ACCÈS À L'ÉDUCATION}

Les femmes rurales au Maroc continuent d'être privées de l'accès à l'éducation puisque le taux d'analphabétisme féminin reste encore élevé. De ce fait, le niveau d'éducation des femmes productrices est très bas.

La capacité des femmes à contribuer à l'utilisation et à la gestion des ressources en eau est en outre entravée par leur manque de connaissances scientifiques et technologiques. Pour faire des choix et prendre des décisions en connaissance de cause, les bénéficiaires potentiels des systèmes d'eau devraient avoir des connaissances de base et une compréhension des technologies concernées. Les femmes sont particulièrement désavantagées en raison des attitudes négatives à l'égard des connaissances techniques féminines. Les femmes ne connaissent ni le prix de l'eau, ni par qui et comment il est calculé. Les femmes adhèrent très peu aux organisations socioprofessionnelles. 


\section{CHÔMAGE CHEZ LES FEMMES}

Les taux de chômage les plus élevés sont enregistrés principalement parmi les femmes (14,7\% contre $8,8 \%$ parmi les hommes), les jeunes âgés de 15 à 24 ans (26,5\% contre 7,7\% parmi les personnes âgées de 25 ans et plus) et les détenteurs d'un diplôme (17,9\% contre 3,8\% parmi les personnes n'ayant aucun diplôme). Ce sont également ces catégories qui ont enregistré les hausses du taux de chômage les plus significatives entre 2016 et 2017 ; 0,6 point parmi les femmes, 0,7 parmi les jeunes de 15-24 ans et 0,3 point parmi les diplômés.

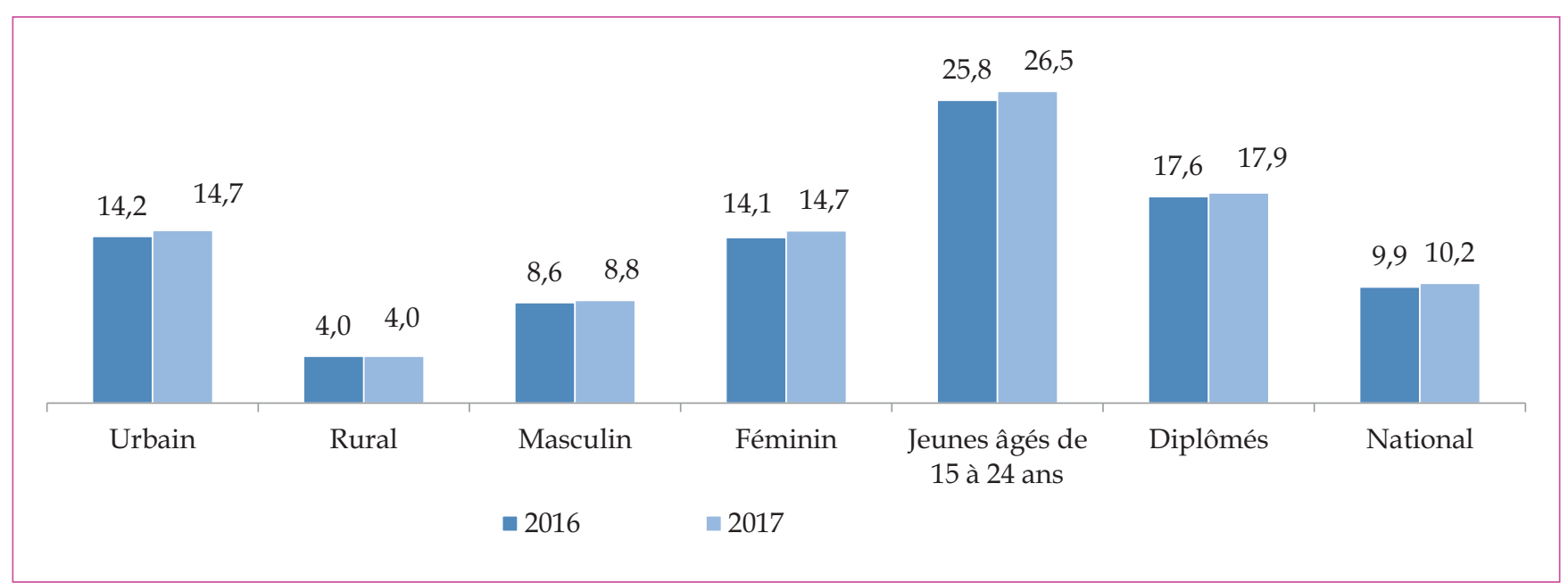

Fig. Evolution du taux de chômage entre 2016 et 2017parmi certaines catégories de la population active(en \%). 


\section{ENGAGEMENTS JURIDIQUES ET POLITIQUES INTERNATIONAUX}

- Reconnaissance de l'importance d'impliquer les femmes et les hommes dans la gestion de l'eau et de l'assainissement [Conférence des Nations Unies sur l'eau de 1977, Mar del Plata; 1981-90 Décennie internationale de l'eau potable et de l'assainissement] ;

- Appel à inclure l'accès des femmes à la prise de décision [Convention sur l'élimination de toutes les formes de discrimination à l'égard des femmes, articles 1 , 2, 3, 4 et 7] ;

- L'intégration de la dimension du genre comme condition préalable à la gestion durable de l'eau [1992, Conférence internationale sur l'eau et l'environnement à Dublin] ;

- Appel à la participation des femmes aux efforts de développement liés à l'eau [Action 21 (paragraphe 18.70f) et le Plan de mise en œuvre de Johannesburg (paragraphe 25); 2005-2015, Décennie internationale d'action, "L'eau, source de vie»] ;

- L'accès à l'eau potable et à l'assainissement en tant que droit de l'homme [Résolution 64/292 de 2010, Assemblée Générale des Nations Unies] ;

- L'importance d'habiliter les femmes rurales en tant qu'agents critiques [2012 Rio + 20 Document finall : 


\section{ENGAGEMENTS JURIDIQUES ET POLITIQUES INTERNATIONAUX}

- Actuellement la plupart des pays ont une législation sur l'égalité des genres.

- Le Maroc est engagé depuis 1956 par des conventions internationales au profit des femmes. Il a ratifié la convention sur l'élimination de toutes formes de discrimination à l'égard des femmes en 1993 et a levé en 2011 ses réserves à cette convention et il l'a adoptée en 2013.

- Souligner les interrelations étroites entre l'égalité des genres et l'autonomisation des femmes [2015 Objectifs de Développement Durable - ODD)] ;

- Concrètement, le Gouvernement marocain a lancé le Plan Gouvernemental pour I'Egalité 2012-2016 "ICRAM 1» et 2017-2021 " ICRAM 2», qui vise la promotion des conditions des femmes marocaines. De plus, la réforme de la Loi Organique 130-13 relative à la Loi de Finances (LOF) constitue une voie de progrès vers l'effectivité de la coordination des politiques publiques. Son principe de base consiste à lier les dépenses publiques aux résultats, dans le but d'évaluer l'efficacité des politiques publiques, en prenant en considération les besoins différenciés des populations cibles afin d'améliorer leur impact, notamment, d'un point de vue genre. 


\section{ENGAGEMENTS JURIDIQUES ET POLITIQUES INTERNATIONAUX}

La nouvelle loi sur l'eau (Loi 36-15) souligne " la prise en compte du droit à l'eau et de l'approche genre dans la gestion des ressources en eau, notamment, à travers la représentation des associations féminines dans les institutions prévues par la loi sur l'eau. ". Elle dit également que " cette loi repose sur des principes fondamentaux parmi lesquels la domanialité publique de l'eau, le droit de tout citoyenne et citoyen à l'accès à l'eau et à un environnement sain, la gestion de l'eau selon les pratiques de bonne gouvernance qui inclut la concertation et la participation de ses différentes parties concernées, la gestion intégrée des ressources en eau en assurant la solidarité spatiale, la protection du milieu naturel et la promotion du développement durable et l'approche genre en matière de développement et de gestion des ressources en eau. ".

Le genre a été pris en compte d'une manière claire dans 4 articles de la loi 36-15 (76, 88 et 89) relatifs au Conseil Supérieur de l'Eau et du Climat, au Conseil des Bassins Hydrauliques et aux Commissions Préfectorales et Provinciales où il est stipulé de réserver $1 / 4$ des représentants des associations œuvrant dans le domaine de l'eau, du climat et de l'environnement pour les femmes. Les décrets d'application de ces conseils et commissions sont déjà approuvés et publiés (décret n².19.205 du 10 juin 2019 concernant les CPP, décret 2.18.233 du 11 juillet 2019 concernant le CSEC et le décret 2.18.768 concernant le CBH du 6 septembre 2019). Pour la police de l'eau, le nombre de ce corps est de 206 à l'échelle nationale dont 23 femmes 


\section{LA CONSTITUTION DU MAROC DE 2011}

- La constitution du Maroc en 2011 a inclus la femme dans :

- Article 19

- L'homme et la femme jouissent, à égalité, des droits et libertés à caractère civil, politique, économique, social, culturel et environnemental,

- L'Etat marocain œuvre à la réalisation de la parité entre les hommes et les femmes. Il est créé, à cet effet, une Autorité pour la parité et la lutte contre toutes formes de discrimination.

- Article 30

- La loi prévoit des dispositions de nature à favoriser l'égal accès des femmes et des hommes aux fonctions électives. Le vote est un droit personnel et un devoir national. Les étrangers jouissent des libertés fondamentales reconnues aux citoyennes et citoyens marocains, conformément à la loi.

- Article 31

- L'Etat, les établissements publics et les collectivités territoriales CEuvrent à la mobilisation de tous les moyens à disposition pour faciliter l'égal accès des citoyennes et des citoyens aux conditions leur permettant de jouir des droits :.

- à l'accès à l'eau et à un environnement sain,

- au développement durable.

- Article 34

- Les pouvoirs publics élaborent et mettent en œuvre des politiques destinées aux personnes et aux catégories à besoins spécifiques. A cet effet, ils veillent notamment à :

- Traiter et prévenir la vulnérabilité de certaines catégories de femmes et de mères, des enfants et des personnes âgées 


\section{EMPLOI CHEZ LES FEMMES}

- Les emplois créés se répartissent entre 57000 emplois rémunérés (22 000 en milieu urbain et 35000 en milieu rural) et 29000 non rémunérés ( 9000 en zones urbaines et 20000 en zones rurales). Ces emplois ont profité à 55 000 hommes et à 31000 femmes.

- Malgré l'accroissement du volume de la population active occupée, le taux d'emploi a baissé de $42,3 \%$ à $41,9 \%(-0,4$ point). Ce taux a également baissé de 0,8 point en milieu urbain et a augmenté de 0,3 point en milieu rural. Entre hommes et femmes, l'écart des taux d'emploi est de 46 points (respectivement 65,4\% et $19,2 \%)$. 


\section{INTÉGRATION GENRE DANS LE DÉPARTEMENT DE L'EAU}

De son côté, le Ministère de l'Equipement, du Transport, de la Logistique et de l'Eau/Département de l'eau est partie prenante du :

- Plan Gouvernemental pour l'Egalité à travers l'institutionnalisation des mécanismes de l'égalité, le renforcement des capacités des intervenants pour intégrer l'approche genre dans les budgets, la fourniture de l'eau potable en milieu rural, et le renforcement de la représentativité des femmes dans les postes de responsabilité, la participation des femmes à la prise de décision, la protection des droits des femmes, la diffusion de la culture de l'égalité et la lutte contre les discriminations et les stéréotypes basés sur le genre, et l'intégration du genre dans la politique, programmes et projets du département de l'eau.

- De ce fait, l'approche genre ne peut plus être une simple option, mais une approche prise en compte dans la politique intégrée des ressources en eau au Maroc. Cela est d'autant plus nécessaire dans un contexte marqué par le changement climatique dont les impacts sur ces ressources en eau affecteront en premier lieu les couches les plus défavorisées. Ces impacts qui se font sentir par la population surtout dans le milieu rural ou leurs effets négatifs rendent leur vécu de plus en plus difficile, surtout pour les femmes et pour les filles. 


\section{INTÉGRATION GENRE DANS LE DÉPARTEMENT DE L'EAU}

Le département de l'eau a élaboré et a démarré la mise en œuvre de sa propre feuille de route pour la question d'adaptation aux changements climatiques avec la considération de l'approche genre. Les priorités qui dominent les actions dans l'intégration de cette approche dans la politique sont :

- l'action de la réorganisation budgétaire annuelle en trois programmes : i) programme météorologie ii) programme pilotage et gouvernance et iii) programme gestion, préservation et développement de l'eau. La budgétisation sensible au genre (BSG) a été intégrée par le département de l'eau depuis 2015 pour les études et l'assistance technique dans le domaine, ce qui a permis la réalisation de l'étude de la stratégie d'institutionnalisation de l'intégration du genre dans le secteur de l'eau et la réalisation des études de cas pour l'identification et l'évaluation des indicateurs genre pour des programmes réalisés par les diverses entités de ce département tel le programme d'approvisionnement en eau potable (AEP) et d'assainissement (ASS) des écoles en milieu rural. 
A noter aussi que la BSG est prise implicitement et visiblement en compte dans les projets d'AEP et d'ASS. L'année 2017 a connu la prise en compte du genre dans des études et projets nécessitant la prise en compte de la population selon le genre (études d'impact sur l'environnement, études d'assainissement en milieu rural ; les études de planification des ressources en eau, l'étude du référentiel des emplois et compétences, les projets d'AEP et d'assainissement en milieu rural, les études et projets de lutte contre les inondations et les projets de mobilisation des ressources en eau de surface, les contrats de nappe ; la communication des prévisions météorologiques; les prévisions météorologiques à court terme ...) ;

- l'action d'institutionnalisation de cette approche à travers l'étude de la Stratégie d'Institutionnalisation de l'Intégration du Genre dans le Secteur de l'Eau (SIIGSE) qui est réalisée dans le cadre de partenariat avec l'ONU Femmes.

L'analyse multidimensionnelle qui a été réalisée dans le cadre de cette étude a fait ressortir les constats et les défis au niveau de toutes les études stratégiques et de planification de l'eau, ainsi qu'au niveau des différents programmes et projets du secteur de l'eau. 


\section{INTÉGRATION GENRE DANS LE DÉPARTEMENT DE L’EAU}

Ceci a permis de ressortir quatre axes stratégiques d'action à savoir : i) le développement d'une capacité institutionnelle pérenne pour assurer l'ancrage de l'égalité de genre dans le secteur de l'eau; ii) l'intégration de l'approche genre dans la gestion des ressources humaines et renforcement de l'égalité des chances pour l'accès des femmes aux postes de responsabilité, iii) l'intégration de l'égalité du genre dans les métiers de l'eau et iv) l'Intégration de l'approche genre dans les programmes et projets réalisés par le département de l'eau avec les partenaires du secteur ;

- I'action de l'intégration du genre dans le plan national de l'eau (PNE), l'une des mesures de la SIIGSE et du Plan gouvernemental pour l'égalité. L'intégration du genre dans le PNE a concerné les axes suivants :

- Gestion de la Demande en Eau améliorée pour les femmes et les hommes ;

- Valorisation des ressources en eau mobilisées ;

- Sauvegarde et pérennisation des barrages ;

- Mobilisation des eaux de surface par les grands barrages;

- Développement local par les petits barrages; 
- Collecte et valorisation des eaux pluviales ; - Transferts d'eau ; - Mobilisation des ressources en eau non conventionnelles ; - Préservation de la qualité des ressources en eau et lutte contre la pollution; - Réduction de la pression sur les surexploitations, pour préserver les ressources en eau souterraine ; Amélioration de l'aménagement et de la protection des bassins versants de manière durable et inclusive ; - Encadrement des processus participatifs et inclusifs pour contribuer à la sauvegarde et la préservation des zones sensibles ; Réduction de la vulnérabilité des femmes et des hommes de toutes catégories, aux risques naturels liés à l'eau et à l'adaptation aux changements climatiques ; Développement de mesures d'accompagnement pour la réalisation de l'intégration de la question du genre dans le PNE;

- l'action de la connaissance de l'approche genre et son intégration dans les politiques publiques sous le prisme droit humain. Cette connaissance a été concrétisée pour les responsables et les cadres du Département de l'eau, des Agences de bassins hydrauliques et de l'Office National de l'Electricité et de I'Eau potable ONEE via des formations et des voyages d'échange au Maroc ou à l'étranger avec le concourt des partenaires étrangers (GIZ et Enabel). Cette action se concrétise chaque année avec les moyens budgétaires et humains propres du département de l'eau. 


\section{INTÉGRATION GENRE DANS LE DÉPARTEMENT DE L’EAU}

\section{Associations féminines}

- Les associations féminines qui œuvrent dans le domaine de l'eau relevant de la zone d'action des $\mathrm{ABHs}$ font partie du deuxième collège constitué des représentants du Conseil des bassins hydrauliques. Les associations féminines œuvrant dans le domaine de l'eau sont aussi représentées dans la Commission Préfectorale ou Provinciale de l'eau. Elles participent donc avec les autres représentants, supervisent et coordonnent la mise en œuvre des actions et mesures entreprises par les services administratifs et communaux pour :

- la gestion de l'eau lors des pénuries d'eau et en cas de force majeure pour assurer l'approvisionnement en eau dans des conditions satisfaisantes ;

- la prévention des risques d'inondation;

- la sensibilisation à la protection des ressources en eau et à la préservation et l'utilisation optimale du domaine public hydraulique;

- La présence de la volonté des pouvoirs publics au profit du genre est prouvée par l'intérêt porté par 31 ministères du pays à ce sujet. II s'agit de l'accès équitable aux droits civils et politiques, aux droits sociaux et au bénéfice équitable des droits économiques. La budgétisation sensible au genre sous le prisme des droits humains a été élaborée dans les stratégies d'actions de tous ces départements ministériels. 


\section{INTÉGRATION GENRE DANS LE DÉPARTEMENT DE L’EAU}

- Le renforcement du rôle de la femme dans la gouvernance du secteur de l'eau est mis en valeur par les efforts déployés par le département de l'eau qui ont permis d'atteindre un taux de $30 \%$ de femmes responsables aux niveaux central, régional et provincial.

\begin{tabular}{|l|l|l|l|}
\hline Grade & H & F & T O T A L \\
\hline Ingénieur général & 4 & 1 & 5 \\
\hline Administrateur & 66 & 57 & 123 \\
\hline Ingénieur en chef & 249 & 49 & 298 \\
\hline Ingénieur d'état & 100 & 94 & 194 \\
\hline Techniciens & 799 & 272 & 1071 \\
\hline Adjoint technique & 635 & 98 & 733 \\
\hline T OT A L & 1853 & 571 & 2424 \\
\hline
\end{tabular}

http://81.192.10.228/wp-content/uploads/2017/03/Integration genre 2019.pdf http://81.192.10.228/le-ministere/ressources-humaines/capital-humain/ 
Le Ministère délégué chargé de l'eau (MDCEau) a adopté l'intégration de l'approche genre en 2014 dans sa politique. De ce fait, un comité genre et une commission technique ont été crées pour la mise en œuvre du plan d'action de la stratégie de l'institutionnalisation de l'intégration du genre dans le secteur de l'eau et des engagements du gouvernement pour instaurer l'égalité et l'équité entre les hommes et les femmes au niveau des programmes et projets en relation avec le secteur de l'eau.

En 2019, un cycle de formation sur l'intégration du genre dans les politiques publiques avec remise des certificats a été organisé par le Ministère délégué chargé de l'eau, en partenariat avec l'Agence Allemande pour la coopération internationale GIZ et l'Agence Belge de développement CTB. II porte sur l'intégration du genre dans les politiques publiques pour renforcer les capacités des cadres du Ministère délégué chargé de l'eau (MDCeau), des Agences de Bassins hydrauliques (ABHs) et de l'Office National de l'Electricité et de l'Eau potable (ONEE), afin de concrétiser I'institutionnalisation du genre dans les divers plans, programmes et projets de ces entités et la diffusion des principes de l'équité et de l'égalité. 
- Le gouvernement marocain a mis en place, en 1995, le Programme d'Approvisionnement Groupe en Eau des Populations Rurales (PAGER). Le but de ce programme était la généralisation de l'accès à l'eau dans de bonnes conditions, permettant à la femme rurale et à ses filles de ne plus s'occuper des tâches d'approvisionnement en eau ; d'augmenter le taux de scolarisation de la fille ; d'améliorer les conditions sanitaires en milieu rural ; de stabiliser la population rurale. Ce projet a augmenté la fréquentation scolaire primaire des écoles rurales (pour les filles et les garçons) de $16 \%$ sur quatre ans. Puisque ces améliorations ont réduit le temps que les filles passaient à transporter de l'eau, elles ont également permis de combler l'écart d'éducation entre les filles et les garçons et les taux de présence des filles ont augmenté de $21 \%$ au cours de la même période (Banque mondiale, 2003). Seulement ce programme n'était pas capable d'assurer un débit régulier surtout en saison sèche. Il faut donc que ces point d'eau soient raccordées à un réseau sûr (barrages).

- Un autre programme d'approvisionnement en eau potable et d'assainissement des écoles rurales (PAEPASER) a concerné 538 écoles, sur la période 2009-2015, réparties sur 56 provinces du Royaume. II tente de faire une évaluation des écoles dépourvues d'installations d'AEP et d'assainissement liquide, ce qui a impacté, certainement, les performances des enseignants et celles des élèves. 